\title{
MODERN FOUNDATIONS FOR STABLE HOMOTOPY THEORY
}

\author{
A. D. ELMENDORF, I. KRIZ, M. A. MANDELL, AND J. P. MAY
}

\section{Contents}

Introduction

1. Spectra and the stable homotopy category 6

2. Smash products and twisted half-smash products 11

3. The category of $\mathbb{L}$-spectra 13

4. The smash product of $\mathbb{L}$-spectra and function $\mathbb{L}$-spectra $\quad 15$

5. The category of $S$-modules 19

6. $S$-algebras and their categories of modules 21

7. The smash product of $R$-modules and function $R$-modules 24

8. Tor and Ext in topology and algebra 28

9. Universal coefficient and Künneth spectral sequences 31

10. Algebraic constructions in the derived category of $R$-modules $\quad 33$

11. $R$-ring structures on localizations and on quotients by ideals $\quad 36$

12. The specialization to $M U$-modules and $M U$-ring spectra 38

$\begin{array}{ll}\text { References } & 40\end{array}$

\section{INTRODUCTION}

It is a truism that algebraic topology is a very young subject. In some of its most fundamental branches, the foundations have not yet reached a state of shared consensus. Our theme will be stable homotopy theory and an emerging consensus on what its foundations should be. The consensus is different than would have been the case as recently as a decade ago. We shall illustrate the force of the change of paradigm with new constructions of some of the most basic objects in modern algebraic topology, namely the various spectra and cohomology theories that can be derived from complex cobordism. The two following articles will give introductions to completions in stable homotopy theory and to equivariant stable homotopy theory. The three papers have a common theme: the relationship between commutative algebra and stable homotopy theory, both relations of analogy and relations of application. 
Stable homotopy theory began around 1937 with the Freudenthal suspension theorem. In simplest terms, it states that, if $q$ is small relative to $n$, then $\pi_{n+q}\left(S^{n}\right)$ is independent of $n$. Stable phenomena had of course appeared earlier, at least implicitly: reduced homology and cohomology are examples of functors that are invariant under suspension without limitation on dimension. Stable homotopy theory emerged as a distinct branch of algebraic topology with Adams' introduction of his eponymous spectral sequence and his spectacular conceptual use of the notion of stable phenomena in his solution to the Hopf invariant one problem. Its centrality was reinforced by two related developments that occurred at very nearly the same time, in the late 1950's. One was the introduction of generalized homology and cohomology theories and especially $K$-theory, by Atiyah and Hirzebruch. The other was the work of Thom which showed how to reduce the problem of classifying manifolds up to cobordism to a problem, more importantly, a solvable problem, in stable homotopy theory.

The reduction of geometric phenomena to solvable problems in stable homotopy theory has remained an important mathematical theme, the most recent major success being Stolz's use of Spin cobordism to study the classification of manifolds with positive scalar curvature. In an entirely different direction, the early 1970's saw Quillen's introduction of higher algebraic $K$-theory and the recognition by Segal and others that it could be viewed as a construction in stable homotopy theory. With algebraic $K$-theory as an intermediary, there has been a growing volume of work that relates algebraic geometry to stable homotopy theory. With Waldhausen's introduction of the algebraic $K$-theory of spaces in the late 1970 's, stable homotopy became a bridge between algebraic $K$-theory and the study of diffeomorphisms of manifolds. Within algebraic topology, the study of stable homotopy theory has been and remains the focus of much of the best work in the subject. The study of nilpotence and periodic phenomena by Hopkins, Mahowald, Ravenel, and many others has been especially successful.

We shall focus on the study of structured ring, module, and algebra spectra. This study plays a significant role in all of the directions of work that we have just mentioned and would have been technically impossible within the foundational consensus that existed a decade ago.

Stable homotopy theory demands a category in which to work. One could set up the ordinary Adams spectral sequence ad hoc, as Adams did, but it would be ugly at best to set up the Adams spectral sequence based on a generalized homology theory that way. One wants objects - called spectra - that play the role of spaces in unstable homotopy theory, and one wants a category in which all of the usual constructions on spaces are present and, up to homotopy, the suspension functor is an equivalence. At this point, we introduce a sharp distinction: there is a category 
of point-set level objects, and there is an associated derived category. There has been consensus on what the latter should be, up to equivalence of categories, since the fundamental work of Boardman in the 1960's. The change in paradigm concerns the point-set level category that underlies the stable homotopy category. There is a growing recognition that one needs a good point-set level category in order to study stable topological algebra seriously.

There is an analogy with algebra that is fundamental to an understanding of this area of mathematics. Suppose given a (discrete) commutative ring $R$. It has

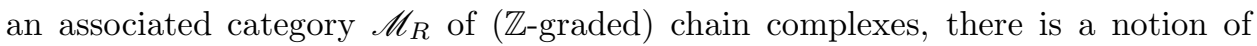
homotopy between maps of chain complexes, and there is a resulting homotopy category $h \mathscr{M}_{R}$. However, this is not the category that algebraists are interested in. For example, if $R$-modules $M$ and $N$ are regarded as chain complexes concentrated in degree zero, then, in the derived category, the homology of their tensor product should be their torsion product $\operatorname{Tor}_{*}^{R}(M, N)$. Formally, the fundamental invariants of chain complexes are their homology groups, and one constructs a category that reflects this. A map of chain complexes is said to be a quasi-isomorphism if it induces an isomorphism of homology groups. The derived category $\mathscr{D}_{R}$ is obtained by adjoining formal inverses to the quasi-isomorphisms. The best way to make this rigorous is to introduce a notion of cell $R$-module such that every quasi-isomorphism between cell $R$-modules is a chain homotopy equivalence (Whitehead theorem) and every chain complex is quasi-isomorphic to a cell $R$-module. Then $\mathscr{D}_{R}$ is equivalent to the ordinary homotopy category of cell $R$-modules. See $[15,21]$. This is a topologist's way of thinking about the appropriate generalization to chain complexes of projective resolutions of modules.

We think of the sphere spectrum $S$ as the analog of $R$. We think of spectra as analogs of chain complexes, or rather as a first approximation to the definitive analogs, which will be $S$-modules. We let $\mathscr{S}$ denote the category of spectra. There is a notion of homotopy of maps between spectra, and there is a resulting homotopy category $h \mathscr{S}$. The fundamental invariants of spectra are their homotopy groups, and a map of spectra is a weak equivalence if it induces an isomorphism of homotopy groups. The stable homotopy category, which we denote by $\bar{h} \mathscr{S}$, is obtained by formally inverting the weak equivalences. This is made rigorous by introducing $\mathrm{CW}$ spectra. A weak equivalence between $\mathrm{CW}$ spectra is a homotopy equivalence and every spectrum is weakly equivalent to a CW spectrum. Then $\bar{h} \mathscr{S}$ is equivalent to the ordinary homotopy category of CW spectra.

Now the category $\mathscr{M}_{R}$ has an associative and commutative tensor product. If we regard $R$ as a chain complex concentrated in degree zero, then $R$ is a unit for the tensor product. A differential $R$-algebra $A$ is a chain complex with a unit $R \longrightarrow A$ and product $A \otimes_{R} A \longrightarrow A$ such that the evident associativity and unity 
diagrams commute. It is commutative if the evident commutativity diagram also commutes. These are, obviously enough, point-set level structures. Algebraists would have trouble taking seriously the idea of an algebra defined in $\mathscr{D}_{R}$, with unit and product only defined in that category.

The category $\mathscr{S}$ has a smash product but, in contrast with the tensor product, it is not associative, commutative, or unital. The induced smash product on the stable homotopy category $\bar{h} \mathscr{S}$ is associative and commutative, and it has $S$ as unit. Topologists routinely study ring spectra, which are objects $E$ of $\bar{h} \mathscr{S}$ with a unit $\eta: S \longrightarrow E$ and product $\phi: E \wedge E \longrightarrow E$ such that the evident unit diagrams commute; that is, $\phi \circ(\eta \wedge \mathrm{id})=\mathrm{id}=\phi \circ(\mathrm{id} \wedge \eta)$ in $\bar{h} \mathscr{S}$. Similarly, $E$ is associative or commutative if the appropriate diagrams commute in $\bar{h} \mathscr{S}$. Given that the point-set level smash product is not associative or commutative, it would seem at first sight that these up to homotopy notions are the only ones possible.

It is a recent discovery that there is a category $\mathscr{M}_{S}$ of $S$-modules that has an associative, commutative, and unital smash product $\wedge_{S}$ [11]. Its objects are spectra with additional structure, and we say that a map of $S$-modules is a weak equivalence if it is a weak equivalence as a map of spectra. The derived category $\mathscr{D}_{S}$ is obtained from $\mathscr{M}_{S}$ by formally inverting the weak equivalences, and $\mathscr{D}_{S}$ is equivalent to the stable homotopy category $\bar{h} \mathscr{S}$. Again, this is made rigorous by a theory of CW $S$-modules that is just like the theory of CW spectra.

In the category $\mathscr{M}_{S}$, we have a point-set level notion of an $S$-algebra $R$ that is defined in terms of maps $\eta: S \longrightarrow R$ and $\phi: R \wedge_{S} R \longrightarrow R$ in $\mathscr{M}_{S}$ such that the standard unit and associativity diagrams commute on the point-set level; we say that $R$ is commutative if the standard commutativity diagram also commutes. There were earlier notions with a similar flavor, namely the $A_{\infty}$ and $E_{\infty}$ ring spectra introduced in $[19,20]$. Here " $A_{\infty}$ " stands historically for "associative up to an infinite sequence of higher homotopies"; similarly, " $E_{\infty}$ " stands for "homotopy everything", meaning that the product is associative and commutative up to all higher coherence homotopies. With the definitions just given, the higher homotopies are hidden in the definition of the associative and commutative smash product in $\mathscr{M}_{S}$, but these definitions are essentially equivalent to the earlier ones, in which the higher homotopies were exhibited in terms of an "operad action". It is tempting to simply call these objects associative and commutative ring spectra, but that would be a mistake. These terms have long established meanings, as associative and commutative rings in the stable homotopy category, and the more precise point-set level notions do not make the older notions obsolete: there are plenty of examples of associative or commutative ring spectra that do not admit structures of $A_{\infty}$ or $E_{\infty}$ ring spectra. It is part of the new paradigm that one must always be aware 
of when one is working in the derived category and when one is working on the point-set level.

Now fix an $S$-algebra $R$. An $R$-module $M$ is an $S$-module together with a map $\mu: R \wedge_{S} M \longrightarrow M$ such that the evident unit and transitivity diagrams commute. Let $\mathscr{M}_{R}$ be the category of $R$-modules. Again we have a homotopy category $h \mathscr{M}_{R}$ and a derived category $\mathscr{D}_{R}$ that is obtained from it by inverting the weak equivalences, by which we mean the maps of $R$-modules that are weak equivalences of underlying spectra. The construction of $\mathscr{D}_{R}$ is made rigorous by a theory of cell $R$-modules, the one slight catch being that, unless $R$ is connective, in the sense that its homotopy groups are zero in negative degrees, we cannot insist that cells be attached only to cells of lower dimension, so that our cell $R$ modules cannot be restricted to be CW $R$-modules. These categories enjoy all of the good properties that we have described in the special case $R=S$. There is an associative, commutative, and unital smash product over $R$. We can therefore go on to define $R$-algebras and commutative $R$-algebras $A$ in terms of point-set level associative, unital, and commutative multiplications $A \wedge_{R} A \longrightarrow A$. We can also define derived category level associative and commutative $R$-ring spectra $A$, exactly like the classical associative and commutative ring spectra in the stable homotopy category.

It is the derived category $\mathscr{D}_{R}$ that we wish to focus on in describing the current state of the art in stable homotopy theory. We can mimic classical commutative algebra in this category. In particular, for an ideal $I$ and multiplicatively closed subset $Y$ in the coefficient ring $R_{*}=\pi_{*}(R)$, we will show how to construct quotients $M / I M$ and localizations $M\left[Y^{-1}\right]$. When applied with $R$ taken to be the representing spectrum $M U$ for complex cobordism, these constructions specialize to give simple constructions of various spectra that are central to modern stable homotopy theory, such as the Morava $K$-theory spectra. Moreover, we shall see that these spectra are $M U$-ring spectra.

This account is largely a summary of parts of the more complete and technical paper [11], to which the reader is referred for further background, detailed proofs, and many more applications. 


\section{Spectra And the Stable homotopy CATEgory}

We here give a bare bones summary of the construction of the stable homotopy category, referring to [16] and [11] for details and to [22] for a more leisurely exposition. We aim to give just enough of the basic definitional framework that the reader can feel comfortable with the ideas.

By Brown's representability theorem [6], if $E^{*}$ is a reduced cohomology theory on based spaces, then there are CW complexes $E_{n}$ such that, for CW complexes $X, E^{n}(X)$ is naturally isomorphic to the set $\left[X, E_{n}\right]$ of homotopy classes of based maps $X \longrightarrow E_{n}$. The suspension isomorphism $E^{n}(X) \cong E^{n+1}(\Sigma X)$ gives rise to a homotopy equivalence $\tilde{\sigma}_{n}: E_{n} \longrightarrow \Omega E_{n+1}$. The object $E=\left\{E_{n}, \tilde{\sigma}_{n}\right\}$ is called an $\Omega$-spectrum. A map $f: E \longrightarrow E^{\prime}$ of $\Omega$-spectra is a sequence of maps $f_{n}: E_{n} \longrightarrow E_{n}^{\prime}$ that are compatible up to homotopy with the equivalences $\tilde{\sigma}_{n}$ and $\tilde{\sigma}_{n}^{\prime}$. The category of $\Omega$-spectra is equivalent to the category of cohomology theories on based spaces and can be thought of as an intuitive first approximation to the stable homotopy category. However, this category does not have a usable theory of cofibration sequences and is not suitable for either point-set level or homotopical work. For that, one needs more precise objects and morphisms that are defined without use of homotopies but that still represent cohomology theories and their maps. More subtly, one needs a coordinate-free setting in order to define smash products sensibly. The $n$th space $E_{n}$ relates to the $n$-sphere and thus to $\mathbb{R}^{n}$. Restricting to spaces $E_{n}$ is very much like restricting to the standard basis of $\mathbb{R}^{\infty}$ when doing linear algebra.

A coordinate-free spectrum is indexed on the set of finite dimensional subspaces $V$ of a "universe" $U$, namely a real inner product space isomorphic to the sum $\mathbb{R}^{\infty}$ of countably many copies of $\mathbb{R}$. In detail, writing $W-V$ for the orthogonal complement of $V$ in $W$, a spectrum $E$ assigns a based space $E V$ to each finite dimensional subspace $V$ of $U$, with (adjoint) structure maps

$$
\tilde{\sigma}_{V, W}: E V \stackrel{\cong}{\longrightarrow} \Omega^{W-V} E W
$$

when $V \subset W$, where $\Omega^{W} X$ is the function space $F\left(S^{W}, X\right)$ of based maps $S^{W} \longrightarrow$ $X$ and $S^{W}$ is the one-point compactification of $W$. The structure maps are required to satisfy an evident transitivity relation when $V \subset W \subset Z$, and they are required to be homeomorphisms. A map of spectra $f: E \rightarrow E^{\prime}$ is a collection of maps of based spaces $f_{V}: E V \rightarrow E^{\prime} V$ for which each of the following diagrams commutes:

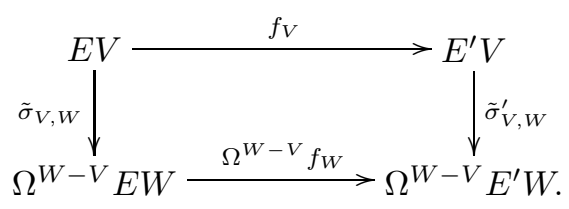


We obtain the category $\mathscr{S}=\mathscr{S} U$ of spectra indexed on $U$. We obtain an equivalent category if we restrict to any cofinal family of indexing spaces. If we drop the requirement that the maps $\tilde{\sigma}_{V, W}$ be homeomorphisms, we obtain the notion of a prespectrum and the category $\mathscr{P}=\mathscr{P} U$ of prespectra indexed on $U$. The forgetful functor $\ell: \mathscr{S} \longrightarrow \mathscr{P}$ has a left adjoint $L$. When the structure maps $\tilde{\sigma}$ are inclusions, $(L E)(V)$ is just the union of the spaces $\Omega^{W-V} E W$ for $V \subset W$. We write $\sigma$ : $\Sigma^{W-V} E V \longrightarrow E W$ for the adjoints of the maps $\tilde{\sigma}$, where $\Sigma^{V} X=X \wedge S^{V}$.

Examples 1.1. Let $X$ be a based space. The suspension prespectrum $\Pi^{\infty} X$ is the prespectrum whose $V$ th space is $\Sigma^{V} X$; the structure maps $\sigma$ are the evident identifications $\Sigma^{W-V} \Sigma^{V} X \cong \Sigma^{W} X$. The suspension spectrum of $X$ is $\Sigma^{\infty} X=$ $L \Pi^{\infty} X$. Let $Q X=\cup \Omega^{V} \Sigma^{V} X$, where the union is taken over the inclusions obtained from the adjoints of the cited identifications. Then $\left(\Sigma^{\infty} X\right)(V)=Q\left(\Sigma^{V} X\right)$. The functor $\Sigma^{\infty}$ from based spaces to spectra is left adjoint to the functor that assigns the zeroth space $E_{0}=E(\{0\})$ to a spectrum $E$. More generally, for a fixed subspace $Z \subset U$, define $\Pi_{Z}^{\infty} X$ to be the analogous prespectrum whose $V$ th space is $\Sigma^{V-Z} X$ if $Z \subset V$ and a point otherwise and define $\Sigma_{Z}^{\infty} X=L \Pi_{Z}^{\infty} X$. Then $\Sigma_{Z}^{\infty}$ is left adjoint to the functor that sends a spectrum to its $Z$ th space $E Z$; these functors are generally called "shift desuspensions".

Functors on prespectra that do not preserve spectra are extended to spectra by applying the functor $L$. For example, for a based space $X$ and a prespectrum $E$, we have the prespectrum $E \wedge X$ specified by $(E \wedge X)(V)=E V \wedge X$. When $E$ is a spectrum, the structure maps for this prespectrum level smash product are not homeomorphisms, and we understand the smash product $E \wedge X$ to be the spectrum $L(\ell E \wedge X)$. Function spectra are easier. We set $F(X, E)(V)=F(X, E V)$ and find that this functor on prespectra preserves spectra. If we topologize the set $\mathscr{S}\left(E, E^{\prime}\right)$ as a subspace of the product over $V$ of the function spaces $F\left(E V, E^{\prime} V\right)$ and let $\mathscr{T}$ be the category of based spaces with sets of maps topologized as function spaces, then there result homeomorphisms

$$
\mathscr{S}\left(E \wedge X, E^{\prime}\right) \cong \mathscr{T}\left(X, \mathscr{S}\left(E, E^{\prime}\right)\right) \cong \mathscr{S}\left(E, F\left(X, E^{\prime}\right)\right) .
$$

Recall that a category is said to be cocomplete if it has all colimits and complete if it has all limits.

Proposition 1.2. The category $\mathscr{S}$ is complete and cocomplete.

Proof. Limits and colimits are defined on prespectra spacewise. Limits preserve spectra, and colimits of spectra are obtained by use of the left adjoint $L$.

We write $Y_{+}$for the union of a space $Y$ and a disjoint basepoint. A homotopy in the category of spectra is a map $E \wedge I_{+} \longrightarrow E^{\prime}$. We have cofibration and fibration 
sequences that are defined exactly as on the space level (e.g. [29]) and enjoy the same homotopical properties. Let $\left[E, E^{\prime}\right]$ denote the set of homotopy classes of maps $E \longrightarrow E^{\prime}$; we shall later understand that, when using this notation, $E$ must be of the homotopy type of a CW spectrum. For based spaces $X$ and $Y$ with $X$ compact, we have

$$
\left[\Sigma^{\infty} X, \Sigma^{\infty} Y\right] \cong \operatorname{colim}_{n}\left[\Sigma^{n} X, \Sigma^{n} Y\right]
$$

Fix a copy of $\mathbb{R}^{\infty}$ in $U$. In the equivariant generalization of the present theory, it is essential not to insist that $\mathbb{R}^{\infty}$ be all of $U$, but the reader may take $U=\mathbb{R}^{\infty}$ here. We write $\Sigma_{n}^{\infty}=\Sigma_{\mathbb{R}^{n}}^{\infty}$. For $n \geq 0$, the sphere spectrum $S^{n}$ is $\Sigma^{\infty} S^{n}$. For $n>0$, the sphere spectrum $S^{-n}$ is $\Sigma_{n}^{\infty} S^{0}$. We write $S$ for the zero sphere spectrum. The $n$th homotopy group of a spectrum $E$ is the set $\left[S^{n}, E\right]$ of homotopy classes of maps $S^{n} \longrightarrow E$, and this fixes the notion of a weak equivalence of spectra. The adjunctions of Examples 1.1 make it clear that a map $f$ of spectra is a weak equivalence if and only if each of its component maps $f_{Z}$ is a weak equivalence of spaces. The stable homotopy category $\bar{h} \mathscr{S}$ is constructed from the homotopy category of spectra by adjoining formal inverses to the weak equivalences, a process that is made rigorous by $\mathrm{CW}$ approximation.

The theory of CW spectra is developed by taking sphere spectra as the domains of attaching maps of cells $C S^{n}=S^{n} \wedge I[16, I \S 5]$. The one major difference from the space level theory of $\mathrm{CW}$ complexes is that we have to construct $\mathrm{CW}$ spectra as unions $E=\cup E_{n}$, where $E_{0}$ is the trivial spectrum and where we are allowed to attach cells of arbitrary dimension when constructing $E_{n+1}$ from $E_{n}$. There results a notion of a cell spectrum. We define a CW spectrum to be a cell spectrum whose cells are attached only to cells of lower dimension. Thus CW spectra have two filtrations, the sequential filtration $\left\{E_{n}\right\}$ that gives the order in which cells are attached, and the skeletal filtration $\left\{E^{q}\right\}$, where $E^{q}$ is the union of the cells of dimension at most $q$. We say that a map between CW spectra is cellular if it preserves both filtrations. In fact, by redefining the sequential filtration appropriately, we can always arrange that the sequential filtration is preserved. We have three basic results, whose proofs are very little different from their space level counterparts.

Theorem 1.3 (Whitehead). If $E$ is a $C W$ spectrum and $f: F \longrightarrow F^{\prime}$ is a weak equivalence of spectra, then $f_{*}:[E, F] \longrightarrow\left[E, F^{\prime}\right]$ is an isomorphism. Therefore a weak equivalence between $C W$ spectra is a homotopy equivalence.

Theorem 1.4 (Cellular approximation). Let $A$ be a subcomplex of a $C W$ spectrum $E$, let $F$ be a $C W$ spectrum, and let $f: E \longrightarrow F$ be a map whose restriction to $A$ is cellular. Then $f$ is homotopic relative to $A$ to a cellular map. Therefore any map 
$E \longrightarrow F$ is homotopic to a cellular map, and any two homotopic cellular maps are cellularly homotopic.

Theorem 1.5 (Approximation by CW spectra). For a spectrum E, there is a $C W$ spectrum $\Gamma E$ and a weak equivalence $\gamma: \Gamma E \longrightarrow E$. On the homotopy category $h \mathscr{S}, \Gamma$ is a functor such that $\gamma$ is natural.

It follows that the stable category $\bar{h} \mathscr{S}$ is equivalent to the homotopy category of $\mathrm{CW}$ spectra. Homotopy-preserving functors on spectra that do not preserve weak equivalences are transported to the stable category by first replacing their variables by weakly equivalent $\mathrm{CW}$ spectra.

Observe that there has been no mention of space level CW complexes in our development so far. The total lack of hypotheses on the spaces and structural maps of our prespectra allows considerable point-set level pathology, even if, as usual in modern algebraic topology, we restrict attention to compactly generated weak Hausdorff spaces. Recall that a space $X$ is weak Hausdorff if the diagonal subspace is closed in the compactly generated product $X \times X$. More restrictively, a space $X$ is said to be LEC (locally equiconnected) if the inclusion of the diagonal subspace is a cofibration. We record the following list of special kinds of prespectra both to prepare for our discussion of smash products and to compare our definitions with those adopted in the original treatments of the stable homotopy category.

Definition 1.6. A prespectrum $D$ is said to be

(i) $\Sigma$-cofibrant if each $\sigma: \Sigma^{W-V} D V \rightarrow D W$ is a based cofibration (that is, satisfies the based homotopy extension property).

(ii) $\mathrm{CW}$ if it is $\Sigma$-cofibrant and each $D V$ is LEC and has the homotopy type of a CW complex.

(iii) strictly $\mathrm{CW}$ if each $D V$ is a based $\mathrm{CW}$ complex and the structure maps $\sigma$ are the inclusions of subcomplexes.

A spectrum $E$ is said to be $\Sigma$-cofibrant if it is isomorphic to $L D$ for some $\Sigma$-cofibrant prespectrum $D ; E$ is said to be tame if it is of the homotopy type of a $\Sigma$-cofibrant spectrum.

If $E$ is a spectrum, then the maps $\tilde{\sigma}$ are homeomorphisms. Therefore the underlying prespectrum $\ell E$ is not $\Sigma$-cofibrant unless it is trivial. However, it is a very weak condition on a spectrum that it be tame. We shall see that this weak condition is enough to avoid serious point-set topological problems. If $D$ is a $\Sigma$-cofibrant prespectrum, then the maps $\tilde{\sigma}$ are inclusions and therefore $L D(V)$ is just the union of the spaces $\Omega^{W-V} D W$. We have the following relations between CW prespectra and CW spectra. Remember that CW spectra are defined in terms of spectrum level attaching maps. 
Theorem 1.7. If $D$ is a $C W$ prespectrum, then $L D$ has the homotopy type of a $C W$ spectrum. If $E$ is a $C W$ spectrum, then each space $E V$ has the homotopy type of a $C W$ complex and $E$ is homotopy equivalent to $L D$ for some $C W$ prespectrum $D$. Thus a spectrum has the homotopy type of a $C W$ spectrum if and only if it has the homotopy type of $L D$ for some $C W$ prespectrum $D$.

In particular, spectra of the homotopy types of CW spectra are tame.

Implicitly or explicitly, early constructions of the stable homotopy category restricted attention to the spectra arising from strict CW prespectra. This is far too restrictive for serious point-set level work, and it is also too restrictive to admit a sensible equivariant analogue. Note that such a category cannot possibly be complete or have well-behaved point-set level function spectra.

One reason for focusing on $\Sigma$-cofibrant spectra is that they are built up out of their component spaces in a simple fashion.

Proposition 1.8. If $E=L D$, where $D$ is a $\Sigma$-cofibrant prespectrum, then

$$
E \cong \operatorname{colim}_{V} \Sigma_{V}^{\infty} D V
$$

where the colimit is computed as the prespectrum level colimit of the maps

$$
\Sigma_{W}^{\infty} \sigma: \Sigma_{V}^{\infty} D V \cong \Sigma_{W}^{\infty} \Sigma^{W-V} D V \longrightarrow \Sigma_{W}^{\infty} D W
$$

That is, the prespectrum level colimit is a spectrum that is isomorphic to E. The maps of the colimit system are shift desuspensions of based cofibrations.

Another reason is that general spectra can be replaced functorially by weakly equivalent $\Sigma$-cofibrant spectra.

Proposition 1.9. There is a functor $K: \mathscr{P} U \longrightarrow \mathscr{P} U$, called the cylinder functor, such that $K D$ is $\Sigma$-cofibrant for any prespectrum $D$, and there is a natural spacewise weak equivalence of prespectra $K D \longrightarrow D$. On spectra $E$, define $K E=L K \ell E$. Then there is a natural weak equivalence of spectra $K E \longrightarrow E$.

In practice, if one is given a prespectrum $D$, perhaps indexed only on integers, and one wishes to construct a spectrum from it that retains homotopical information, one forms $E=L K D$. Then

$$
\pi_{n}(E)=\operatorname{colim}_{q} \pi_{n+q} D_{q}
$$

If $D$ is an $\Omega$-spectrum that represents a given cohomology theory on spaces, then $E=L K D$ is a genuine spectrum that represents the same theory. 


\section{SMASH PRODUCTS AND TWISTED HALF-SMASH PRODUCTS}

The construction of the smash product of spectra proceeds by internalization of an "external smash product". The latter is an associative and commutative pairing

$$
\mathscr{S} U \times \mathscr{S} U^{\prime} \rightarrow \mathscr{S}\left(U \oplus U^{\prime}\right)
$$

for any pair of universes $U$ and $U^{\prime}$. It is constructed by starting with the prespectrum level definition

$$
\left(E \wedge E^{\prime}\right)\left(V \oplus V^{\prime}\right)=E V \wedge E^{\prime} V^{\prime} .
$$

The structure maps fail to be homeomorphisms when $E$ and $E^{\prime}$ are spectra, and we apply the spectrification functor $L$ to obtain the desired spectrum level smash product.

In order to obtain smash products internal to a single universe $U$, we exploit the "twisted half-smash product". The input data for this functor consist of two universes $U$ and $U^{\prime}$, an unbased space $A$ with a given map $\alpha: A \rightarrow \mathscr{I}\left(U, U^{\prime}\right)$, and a spectrum $E$ indexed on $U$. The output is the spectrum $A \ltimes E$, which is indexed on $U^{\prime}$. It must be remembered that the construction depends on $\alpha$ and not just on $A$, although different choices of $\alpha$ lead to equivalent functors on the level of stable categories. When $A$ is a point, $\alpha$ is a choice of a linear isometry $f: U \longrightarrow U^{\prime}$ and we write $f_{*}$ for the twisted half-smash product. For a prespectrum $D$,

$$
\left(f_{*} D\right)\left(V^{\prime}\right)=D(V) \wedge S^{V^{\prime}-f(V)} \text {, where } V=f^{-1}\left(V^{\prime} \cap \operatorname{im} f\right) .
$$

For a spectrum $E, f_{*} E$ is obtained by application of $L$ to the prespectrum level construction. The functor $f_{*}$ is left adjoint to the more elementary functor $f^{*}$ specified by $\left(f^{*} E^{\prime}\right)(V)=E^{\prime}(f(V))$. For general $A$ and $\alpha$, the intuition is that $A \ltimes E$ is obtained by suitably topologizing the union of the $\alpha(a)_{*}(E)$. Another intuition is that the twisted half-smash product is a generalization to spectra of the "untwisted" functor $A_{+} \wedge X$ on based spaces $X$. This intuition is made precise by the following "untwisting formula" relating twisted half-smash products and shift desuspensions.

Proposition 2.1. For a map $A \longrightarrow \mathscr{I}\left(U, U^{\prime}\right)$ and an isomorphism $V \cong V^{\prime}$, where $V \subset U$ and $V^{\prime} \subset U^{\prime}$, there is an isomorphism of spectra

$$
A \ltimes \Sigma_{V}^{\infty} X \cong A_{+} \wedge \Sigma_{V^{\prime}}^{\infty} X
$$

that is natural in spaces $A$ over $\mathscr{I}\left(U, U^{\prime}\right)$ and based spaces $X$.

The twisted-half smash product functor enjoys essentially the same formal properties as the space level functor $A_{+} \wedge X$. The functor $A \ltimes E$ is homotopy-preserving in $E$, and it therefore preserves homotopy equivalences in the variable $E$. However, it only preserves homotopies over $\mathscr{I}\left(U, U^{\prime}\right)$ in $A$. Nevertheless, it very often 
preserves homotopy equivalences in the variable $A$. The following central technical result is an easy consequence of Propositions 1.8 and 2.1 .

Theorem 2.2. Let $E \in \mathscr{S} U$ be tame and let $A$ be a space over $\mathscr{I}\left(U, U^{\prime}\right)$. If $\phi: A^{\prime} \longrightarrow A$ is a homotopy equivalence, then $\phi \ltimes$ id $: A^{\prime} \ltimes E \longrightarrow A \ltimes E$ is a homotopy equivalence.

Since $A \ltimes E$ is a $\mathrm{CW}$ spectrum if $A$ is a CW complex and $E$ is a $\mathrm{CW}$ spectrum, this has the following consequence.

Corollary 2.3. Let $E \in \mathscr{S} U$ be a spectrum that has the homotopy type of a $C W$ spectrum and let $A$ be a space over $\mathscr{I}\left(U, U^{\prime}\right)$ that has the homotopy type of a $C W$ complex. Then $A \ltimes E$ has the homotopy type of a $C W$ spectrum.

Now, as before, restrict attention to a particular universe $U$ and write $\mathscr{S}=\mathscr{S} U$; again, the reader may think of $U$ as $\mathbb{R}^{\infty}$. We are especially interested in twisted half-smash products defined in terms of the following spaces of linear isometries.

Notations 2.4. Let $U^{j}$ be the direct sum of $j$ copies of $U$ and let $\mathscr{L}(j)=\mathscr{I}\left(U^{j}, U\right)$. The space $\mathscr{L}(0)$ is the point $i$, where $i:\{0\} \rightarrow U$, and $\mathscr{L}(1)$ contains the identity map $1=\operatorname{id}_{U}: U \rightarrow U$. The left action of $\Sigma_{j}$ on $U^{j}$ by permutations induces a free right action of $\Sigma_{j}$ on the contractible space $\mathscr{L}(j)$. Define maps

$$
\gamma: \mathscr{L}(k) \times \mathscr{L}\left(j_{1}\right) \times \cdots \times \mathscr{L}\left(j_{k}\right) \longrightarrow \mathscr{L}\left(j_{1}+\cdots+j_{k}\right)
$$

by

$$
\gamma\left(g ; f_{1}, \ldots, f_{k}\right)=g \circ\left(f_{1} \oplus \cdots \oplus f_{k}\right) .
$$

The spaces $\mathscr{L}(j)$ form an operad [18, p.1] with structural maps $\gamma$, called the linear isometries operad. Points $f \in \mathscr{L}(j)$ give functors $f_{*}$ that send spectra indexed on $U^{j}$ to spectra indexed on $U$. Applied to a $j$-fold external smash product $E_{1} \wedge \cdots \wedge E_{j}$, there results an internal smash product $f_{*}\left(E_{1} \wedge \cdots \wedge E_{j}\right)$. All of these smash products become equivalent in the stable category $\bar{h} \mathscr{S}$, but none of them are associative or commutative on the point set level. More precisely, the following result holds.

Theorem 2.5. Let $\mathscr{S}_{t} \subset \mathscr{S}$ be the full subcategory of tame spectra and let $h \mathscr{S}_{t}$ be its homotopy category. On $\mathscr{S}_{t}$, the internal smash products $f_{*}\left(E \wedge E^{\prime}\right)$ determined by varying $f \in \mathscr{L}(2)$ are canonically homotopy equivalent, and $h \mathscr{S}_{t}$ is symmetric monoidal under the internal smash product. For based spaces $X$ and tame spectra $E$, there is a natural homotopy equivalence $E \wedge X \simeq f_{*}\left(E \wedge \Sigma^{\infty} X\right)$.

This implies formally that we have arrived at a stable situation. As for spaces, the suspension functor $\Sigma$ is given by $\Sigma E=E \wedge S^{1}$ and is left adjoint to the loop functor $\Omega$ given by $\Omega E=F\left(S^{1}, E\right)$. The cofibre $C f$ of a map $f: E \longrightarrow E^{\prime}$ of spectra is the pushout $E^{\prime} \cup_{f} C E$. 
Theorem 2.6. The suspension functor $\Sigma: h \mathscr{S}_{t} \longrightarrow h \mathscr{S}_{t}$ is an equivalence of categories. A cofibre sequence $E \stackrel{f}{\longrightarrow} E^{\prime} \longrightarrow C f$ in $\mathscr{S}_{t}$ gives rise to a long exact sequence of homotopy groups

$$
\cdots \longrightarrow \pi_{q}(E) \longrightarrow \pi_{q}\left(E^{\prime}\right) \longrightarrow \pi_{q}(C f) \longrightarrow \pi_{q-1}(E) \longrightarrow \cdots .
$$

Proof. For based spaces $X, \Sigma^{\infty} X$ is naturally isomorphic to $\left(\Sigma_{1}^{\infty} X\right) \wedge S^{1}$ because the structural homeomorphisms $\tilde{\sigma}: E_{0} \longrightarrow \Omega E_{1}$ on spectra give an isomorphism between their right adjoints. Thus, for tame spectra $E$, the previous theorem gives a natural homotopy equivalence

$$
E=E \wedge S^{0} \simeq f_{*}\left(E \wedge \Sigma^{\infty} S^{0}\right) \cong f_{*}\left(E \wedge \Sigma_{1}^{\infty} S^{0}\right) \wedge S^{1} .
$$

Therefore $\Sigma$ is an equivalence of categories with inverse obtained by smashing with the (-1)-sphere spectrum $S^{-1}=\Sigma_{1}^{\infty} S^{0}$. It follows categorically that $\Omega E \simeq f_{*}(E \wedge$ $S^{-1}$ ) and that the unit and counit

$$
\eta: E \longrightarrow \Omega \Sigma E \quad \text { and } \quad \varepsilon: \Sigma \Omega E \longrightarrow E
$$

of the adjunction are homotopy equivalences. The last statement is a standard consequence of the fact that maps can now be desuspended.

Note that only actual homotopy equivalences, not weak ones, are relevant to the last two results. For this reason among others, $h \mathscr{S}_{t}$ is a technically convenient halfway house between the homotopy category of spectra and the stable homotopy category.

\section{The CATEGory of $\mathbb{L}$-SPECTRA}

We need a category of spectra with a canonical smash product. The category of $\mathbb{L}$-spectra that we introduce here will be shown in the next section to have an associative and commutative smash product $\wedge \mathscr{L}$. This product is not quite unital, but there is a natural unit weak equivalence $\lambda: S \wedge \mathscr{L} M \longrightarrow M$. The $S$-modules will be the $\mathbb{L}$-spectra such that $\lambda$ is an isomorphism.

For $f \in \mathscr{L}(j)$ and $E_{i} \in \mathscr{S}_{t}$, Theorem 2.2 implies that the inclusion $\{f\} \subset \mathscr{L}(j)$ induces a homotopy equivalence

$$
f_{*}\left(E_{1} \wedge \cdots \wedge E_{j}\right) \longrightarrow \mathscr{L}(j) \ltimes\left(E_{1} \wedge \cdots \wedge E_{j}\right) .
$$

The proof of Theorem 2.5 above is entirely based on the use of such equivalences. It therefore seems natural to think of

$$
\mathscr{L}(j) \ltimes\left(E_{1} \wedge \cdots \wedge E_{j}\right)
$$

as a canonical $j$-fold smash product. It is still not associative, but it seems closer to being so. However, to take this idea seriously, we must take note of the difference between $E$ and its "1-fold smash product" $\mathscr{L}(1) \ltimes E$. The space $\mathscr{L}(1)$ is a monoid 
under composition, and the formal properties of twisted half-smash products imply a natural isomorphism

$$
\mathscr{L}(1) \ltimes(\mathscr{L}(1) \ltimes E) \cong(\mathscr{L}(1) \times \mathscr{L}(1)) \ltimes E,
$$

where, on the right, $\mathscr{L}(1) \times \mathscr{L}(1)$ is regarded as a space over $\mathscr{L}(1)$ via the composition product. This product induces a map $\mu:(\mathscr{L}(1) \times \mathscr{L}(1)) \ltimes E \longrightarrow \mathscr{L}(1) \ltimes E$, and the inclusion $\{1\} \longrightarrow \mathscr{L}(1)$ induces a map $\eta: E \longrightarrow \mathscr{L}(1) \ltimes E$. Thus it makes sense to consider spectra $E$ with an action $\xi: \mathscr{L}(1) \ltimes E \longrightarrow E$ of the monoid $\mathscr{L}(1)$. It is required that the following diagrams commute:

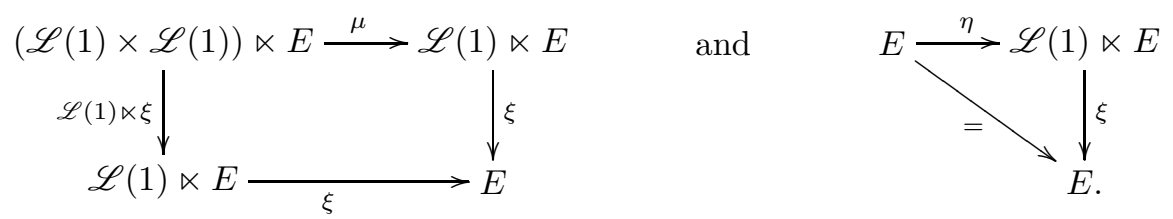

Definition 3.1. An $\mathbb{L}$-spectrum is a spectrum $E$ together with an action $\xi$ of $\mathscr{L}(1)$. A map $f: E \rightarrow E^{\prime}$ of $\mathbb{L}$-spectra is a map of spectra such that the following diagram commutes:

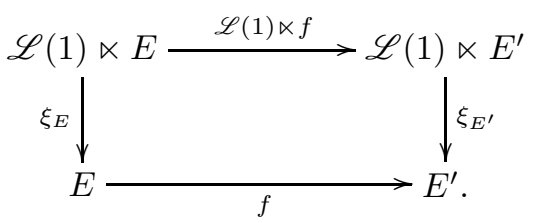

We let $\mathscr{S}[\mathbb{L}]$ denote the category of $\mathbb{L}$-spectra.

A number of basic properties of the category of spectra are directly inherited by the category of $\mathbb{L}$-spectra.

Theorem 3.2. The category of $\mathbb{L}$-spectra is complete and cocomplete, with both limits and colimits created in the underlying category $\mathscr{S}$. If $X$ is a based space and $M$ is an $\mathbb{L}$-spectrum, then $M \wedge X$ and $F(X, M)$ are $\mathbb{L}$-spectra, and the spectrum level fibre and cofibre of a map of $\mathbb{L}$-spectra are $\mathbb{L}$-spectra.

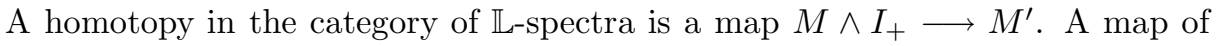
$\mathbb{L}$-spectra is a weak equivalence if it is a weak equivalence as a map of spectra. The stable homotopy category $\bar{h} \mathscr{S}[\mathbb{L}]$ is constructed from the homotopy category of $\mathbb{L}$-spectra by adjoining formal inverses to the weak equivalences. There is a theory of $\mathrm{CW} \mathbb{L}$-spectra that is exactly like the theory of CW spectra, and, again, the construction of $\bar{h} \mathscr{S}[\mathbb{L}]$ is made rigorous by $\mathrm{CW}$ approximation. We have a free functor $\mathbb{L}$ from spectra to $\mathbb{L}$-spectra specified by $\mathbb{L} E=\mathscr{L}(1) \ltimes E$. The "sphere $\mathbb{L}$-spectra" that we take as the domains of attaching maps when defining $\mathrm{CW} \mathbb{L}$ spectra are the free $\mathbb{L}$-spectra $\mathbb{L} S^{n}$. Using the freeness adjunction

$$
\mathscr{S}[\mathbb{L}](\mathbb{L} E, M) \cong \mathscr{S}(E, M)
$$


it is easy to prove Whitehead, cellular approximation, and approximation by CW $\mathbb{L}$-spectra theorems exactly like those stated for spectra in Section 1, and $\bar{h} \mathscr{S}[\mathbb{L}]$ is equivalent to the homotopy category of $\mathrm{CW} \mathbb{L}$-spectra. There is one catch: although $S$ and all other suspension spectra are $\mathbb{L}$-spectra in a natural way, using the untwisting isomorphism of Proposition 2.1 and the projection $\mathscr{L}(1) \longrightarrow\{*\}, S$ does not have the homotopy type of a $\mathrm{CW} \mathbb{L}$-spectrum. However, it is not hard to see that the categories $\bar{h} \mathscr{S}$ and $\bar{h} \mathscr{S}[\mathbb{L}]$ are equivalent.

Theorem 3.3. The following conclusions hold.

(i) The free functor $\mathbb{L}: \mathscr{S} \longrightarrow \mathscr{S}[\mathbb{L}]$ carries $C W$ spectra to $C W \mathbb{L}$-spectra.

(ii) The forgetful functor $\mathscr{S}[\mathbb{L}] \longrightarrow \mathscr{S}$ carries $\mathbb{L}$-spectra of the homotopy types of $C W \mathbb{L}$-spectra to spectra of the homotopy types of $C W$ spectra.

(iii) Every $C W \mathbb{L}$-spectrum $M$ is homotopy equivalent as an $\mathbb{L}$-spectrum to $\mathbb{L} E$ for some $C W$ spectrum $E$.

(iv) If $E \in \mathscr{S}_{t}$, for example if $E$ is a $C W$ spectrum, then $\eta: E \longrightarrow \mathbb{L} E$ is a homotopy equivalence of spectra.

(v) If $M$ has the homotopy type of a $C W \mathbb{L}$-spectrum, then $\xi: \mathbb{L} M \longrightarrow M$ is a homotopy equivalence of $\mathbb{L}$-spectra.

Therefore the free and forgetful functors establish an adjoint equivalence between the stable homotopy categories $\bar{h} \mathscr{S}$ and $\bar{h} \mathscr{S}[\mathbb{L}]$.

\section{The SMASh PRODUCT of $\mathbb{L}$-SPECTRA AND FUnCTION $\mathbb{L}$-SPECTRA}

One of the most surprising developments of recent years is the discovery of an associative and commutative smash product $\wedge_{\mathscr{L}}$ in the category of $\mathbb{L}$-spectra. We proceed to define it. To begin with, observe that the monoid $\mathscr{L}(1) \times \mathscr{L}(1)$ acts from the right on $\mathscr{L}(2)$ and acts from the left on $\mathscr{L}(i) \times \mathscr{L}(j)$, via instances of the structural maps $\gamma$ of the linear isometries operad. Another instance of $\gamma$ gives rise to a map

$$
\bar{\gamma}: \mathscr{L}(2) \times \mathscr{L}(1) \times \mathscr{L}(1) \mathscr{L}(i) \times \mathscr{L}(j) \longrightarrow \mathscr{L}(i+j) .
$$

The space on the left is the balanced product (formally a coequalizer) of the two specified actions by $\mathscr{L}(1) \times \mathscr{L}(1)$. The essential, elementary, point is that this map is a homeomorphism if $i \geq 1$ and $j \geq 1$. To see this, choose linear isometric isomorphisms $s: U \longrightarrow U^{i}$ and $t: U \longrightarrow U^{j}$. Composition on the right with $s \oplus t$ gives vertical homeomorphisms in the commutative diagram

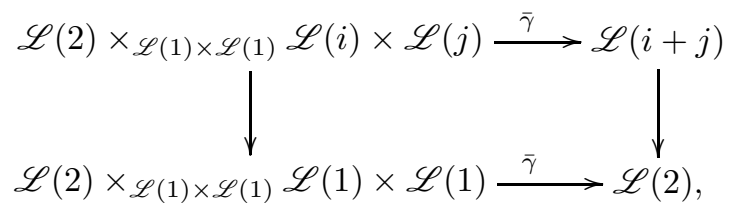


and the lower map $\bar{\gamma}$ is clearly a homeomorphism. Note also that $\mathscr{L}(1)$ acts from the left on $\mathscr{L}(2)$ and that this action commutes with the right action of $\mathscr{L}(1) \times \mathscr{L}(1)$.

Regard $\mathscr{L}(1) \times \mathscr{L}(1)$ as a space over $\mathscr{I}\left(U^{2}, U^{2}\right)$ via the direct sum of isometries map. If $M$ and $N$ are $\mathbb{L}$-spectra, then $\mathscr{L}(1) \times \mathscr{L}(1)$ acts from the left on the external smash product $M \wedge N$ via the map

$$
\xi:(\mathscr{L}(1) \times \mathscr{L}(1)) \ltimes(M \wedge N) \cong(\mathscr{L}(1) \ltimes M) \wedge(\mathscr{L}(1) \ltimes N) \stackrel{\xi \wedge \xi}{\longrightarrow} M \wedge N .
$$

The operadic smash product of $M$ and $N$ is simply the balanced product (again, formally a coequalizer)

$$
M \wedge \mathscr{L} N=\mathscr{L}(2) \ltimes \mathscr{L}(1) \times \mathscr{L}(1)(M \wedge N) .
$$

The left action of $\mathscr{L}(1)$ on $\mathscr{L}(2)$ induces a left action of $\mathscr{L}(1)$ on $M \wedge \mathscr{L} N$ that gives it a structure of $\mathbb{L}$-spectrum. Use of the transposition $\sigma \in \Sigma_{2}$ and the commutativity of the external smash product easily gives a commutativity isomorphism

$$
\tau: M \wedge_{\mathscr{L}} N \longrightarrow N \wedge_{\mathscr{L}} M
$$

More substantially, there is a natural associativity isomorphism

$$
(M \wedge \mathscr{L} N) \wedge \mathscr{L} P \cong M \wedge_{S}(N \wedge \mathscr{L} P) .
$$

In fact, using the case $i=2$ and $j=1$ of the homeomorphism $\bar{\gamma}$, we obtain isomorphisms

$$
\begin{aligned}
(M \wedge \mathscr{L} N) \wedge \mathscr{L} P & \cong \mathscr{L}(2) \ltimes \mathscr{L}(1)^{2}\left(\mathscr{L}(2) \ltimes \mathscr{L}(1)^{2}(M \wedge N)\right) \wedge(\mathscr{L}(1) \ltimes \mathscr{L}(1) P) \\
& \cong\left(\mathscr{L}(2) \times \mathscr{L}(1)^{2} \mathscr{L}(2) \times \mathscr{L}(1)\right) \ltimes \mathscr{L}(1)^{3}(M \wedge N \wedge P) \\
& \cong \mathscr{L}(3) \ltimes \mathscr{L}(1)^{3} M \wedge N \wedge P .
\end{aligned}
$$

The symmetric argument shows that this is also isomorphic to $M \wedge \mathscr{L}(N \wedge \mathscr{L} P)$. In view of the generality of the homeomorphisms (4.1), the argument iterates to give

$$
M_{1} \wedge \mathscr{L} \cdots \wedge \mathscr{L} M_{j} \cong \mathscr{L}(j) \ltimes \mathscr{L}(1)^{j}\left(M_{1} \wedge \cdots \wedge M_{j}\right),
$$

where the iterated smash product on the left is associated in any fashion.

On passage to the derived category $\bar{h} \mathscr{S}[\mathbb{L}]$, the smash product of $\mathbb{L}$-spectra just constructed can be used interchangeably with the internal smash product on the stable category $\bar{h} \mathscr{S}$. To see this, one defines the latter by use of a linear isometric isomorphism $f: U^{2} \longrightarrow U$ (not just an isometry). With this choice, it is not hard to check the following result.

Proposition 4.1. For spectra $E$ and $F$, there are isomorphisms of $\mathbb{L}$-spectra

$$
\mathbb{L} E \wedge \mathscr{L} \mathbb{L} F \cong \mathscr{L}(2) \ltimes E \wedge F \cong \mathbb{L} f_{*}(E \wedge F) .
$$

For $C W \mathbb{L}$-spectra $M$ and $N, M \wedge \mathscr{L} N$ is a $C W \mathbb{L}$-spectrum with one $(p+q)$-cell for each $p$-cell of $M$ and q-cell of $N$. 
However, we need a deeper result, one that depends on the fine structure of the linear isometries operad, to complete the comparison of smash products. By arguments like those in the proof of Theorem 2.6, its first statement implies its second statement.

Proposition 4.2. For $\mathbb{L}$-spectra $N$, there is a natural weak equivalence of $\mathbb{L}$-spectra $\omega: \mathbb{L} S \wedge \mathscr{L} N \longrightarrow N$, and $\Sigma: \pi_{n}(N) \longrightarrow \pi_{n+1}(\Sigma N)$ is an isomorphism for all integers $n$. Therefore the unit $\eta: N \longrightarrow \Omega \Sigma N$ and counit $\varepsilon: \Sigma \Omega N \longrightarrow N$ of the $(\Sigma, \Omega)$-adjunction are weak equivalences and any cofibre sequence $N \stackrel{f}{\longrightarrow} N^{\prime} \longrightarrow C f$ of $\mathbb{L}$-spectra gives rise to a long exact sequence of homotopy groups

$$
\cdots \longrightarrow \pi_{q}(N) \longrightarrow \pi_{q}\left(N^{\prime}\right) \longrightarrow \pi_{q}(C f) \longrightarrow \pi_{q-1}(N) \longrightarrow \cdots .
$$

It is a pleasant technical feature of the theory that this result holds whether or not the given $\mathscr{L}$-spectra are tame. In particular, we have the following consequence, which is the $\mathbb{L}$-spectrum analog of the algebraic statement that, when computing torsion products, one need only resolve one of the tensor factors by a projective resolution.

Proposition 4.3. If $M$ is a $C W \mathbb{L}$-spectrum and $\phi: N \longrightarrow N^{\prime}$ is a weak equivalence of $\mathbb{L}$-spectra, then id $\wedge \mathscr{L} \phi: M \wedge \mathscr{L} N \longrightarrow M \wedge_{\mathscr{L}} N^{\prime}$ is a weak equivalence of $\mathbb{L}$-spectra.

The previous results lead easily to the promised comparison between the internal smash product of spectra and the operadic smash product of $\mathbb{L}$-spectra.

Theorem 4.4. For $\mathbb{L}$-spectra $M$ and $N$, there is a natural map of spectra

$$
\alpha: f_{*}(M \wedge N) \longrightarrow M \wedge \mathscr{L} N,
$$

and $\alpha$ is a weak equivalence when $M$ is a $C W \mathbb{L}$-spectrum and $N$ is a tame spectrum. For any $\mathbb{L}$-spectrum $N$, the functors $(?) \wedge \mathscr{L} N$ and $f_{*}(? \wedge N)$ from $\bar{h} \mathscr{S}[\mathbb{L}]$ to $\bar{h} \mathscr{S}$ are naturally isomorphic.

Thus, under the forgetful functor, the operadic smash product in $\bar{h} \mathscr{S}[\mathbb{L}]$ agrees with the internal smash product in $\bar{h} \mathscr{S}$.

There is a function $\mathbb{L}$-spectrum functor to go with the operad smash product. The twisted half-smash product functor $A \ltimes E$ has a right adjoint twisted function spectrum functor $F\left[A, E^{\prime}\right)$ and the external smash product has a right adjoint function spectrum functor. Using these functors and appropriate equalizer diagrams, dual to the coequalizer diagrams that were implicit in the definition of $\wedge \mathscr{L}$, we obtain the following result.

Theorem 4.5. There is a function $\mathbb{L}$-spectrum functor $F_{\mathscr{L}}(M, N)$ such that

$$
\mathscr{S}[\mathbb{L}](M \wedge \mathscr{L} N, P) \cong \mathscr{S}[\mathbb{L}]\left(M, F_{\mathscr{L}}(N, P)\right)
$$


for $\mathbb{L}$-spectra $M, N$, and $P$.

Given the adjunction, we can deduce the homotopical behavior of $F_{\mathscr{L}}$ from that of $\wedge \mathscr{L}$. There is an internal function spectrum functor $F$ that is induced from the external spectrum functor by use of our chosen linear isometric isomorphism $f: U^{2} \longrightarrow U$. Our function $\mathscr{L}$-spectrum functor gives a canonical substitute.

Proposition 4.6. If $M$ is a $C W \mathbb{L}$-spectrum and $\phi: N \rightarrow N^{\prime}$ is a weak equivalence of $\mathbb{L}$-spectra, then

$$
F_{\mathscr{L}}(\mathrm{id}, \phi): F_{\mathscr{L}}(M, N) \longrightarrow F_{\mathscr{L}}\left(M, N^{\prime}\right)
$$

is a weak equivalence of $\mathbb{L}$-spectra.

Theorem 4.7. For $\mathbb{L}$-spectra $M$ and $N$, there is a natural map of spectra

$$
\tilde{\alpha}: F_{\mathscr{L}}(M, N) \longrightarrow F\left(M, f^{*} N\right),
$$

and $\tilde{\alpha}$ is a weak equivalence when $M$ is a $C W \mathbb{L}$-spectrum. The forgetful functor $\bar{h} \mathscr{S}[\mathbb{L}] \longrightarrow \bar{h} \mathscr{S}$ carries the function $\mathbb{L}$-spectrum functor $F_{\mathscr{L}}$ to the internal function spectrum functor $F$.

We must still address the question of units.

Proposition 4.8. For $\mathbb{L}$-spectra $N$, there is a natural unit map of $\mathbb{L}$-spectra $\lambda$ : $S \wedge \mathscr{L} N \longrightarrow N$. It is a weak equivalence for any $N$, and it is a homotopy equivalence of $\mathbb{L}$-spectra if $N$ is a $C W \mathbb{L}$-spectrum.

Proof. Consider the map $\bar{\gamma}$ of (4.1). It is a non-trivial property of the linear isometries operad that $\bar{\gamma}$, although not a homeomorphism, is a homotopy equivalence when $i=0$ and $j>0$. When $N$ is the free $S$-module $\mathbb{L} E=\mathscr{L}(1) \ltimes E$ generated by a spectrum $E, \lambda$ is given by the map

$$
\begin{aligned}
S \wedge_{S} \mathbb{L} E= & \mathscr{L}(2) \ltimes \mathscr{L}(1) \times \mathscr{L}(1)\left(\mathscr{L}(0) \ltimes S^{0}\right) \wedge(\mathscr{L}(1) \ltimes E) \\
& \cong(\mathscr{L}(2) \times \mathscr{L}(1) \times \mathscr{L}(1) \\
& \stackrel{\bar{\gamma} \ltimes \mathrm{iid}}{\longrightarrow} \mathscr{L}(1) \ltimes E=\mathbb{L} E .
\end{aligned}
$$

Since $\bar{\gamma}$ is a homotopy equivalence, Theorem 2.2 implies that $\lambda$ is a homotopy equivalence when $E \in \mathscr{S}_{t}$. For general $N$, the map just constructed for $\mathbb{L} N$ induces the required map for $N$ by a comparison of coequalizer diagrams. Although the arguments are not transparent, the rest can be deduced from this.

There is one important case when $\lambda$ is an isomorphism. It turns out that the map $\bar{\gamma}$ of (4.1) is a homeomorphism when $i=j=0$; that is, non-obviously since $\mathscr{L}(1)$ is a monoid but not a group, the domain $\mathscr{L}(2) / \mathscr{L}(1) \times \mathscr{L}(1)$ of $(4.1)$ is then a point. This implies that $S \wedge \mathscr{L} S \cong S$. More generally, it implies that the smash 
product over $\mathscr{L}$ precisely generalizes the smash product of based spaces, in the sense that

$$
\Sigma^{\infty} X \wedge \mathscr{L} \Sigma^{\infty} Y \cong \Sigma^{\infty}(X \wedge Y)
$$

\section{The CATEgory of $S$-Modules}

Here, finally, is the promised definition of $S$-modules.

Definition 5.1. Define an $S$-module to be an $\mathbb{L}$-spectrum $M$ which is unital in the sense that $\lambda: S \wedge \mathscr{L} M \longrightarrow M$ is an isomorphism. Let $\mathscr{M}_{S}$ denote the full subcategory of $\mathscr{S}[\mathbb{L}]$ whose objects are the $S$-modules. For $S$-modules $M$ and $N$, define

$$
M \wedge_{S} N=M \wedge_{\mathscr{L}} N \quad \text { and } \quad F_{S}(M, N)=S \wedge_{\mathscr{L}} F_{\mathscr{L}}(M, N) .
$$

The justification for the name " $S$-module" is given by the commutative diagrams

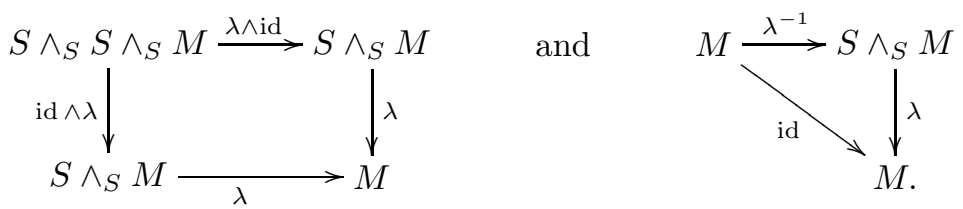

We consistently retain the notation $M \wedge_{\mathscr{L}} N$ when the given $\mathbb{L}$-spectra $M$ and $N$ are not restricted to be $S$-modules. We have the following examples of $S$-modules.

Proposition 5.2. For any based space $X, \Sigma^{\infty} X$ is an $S$-module, and

$$
\Sigma^{\infty} X \wedge_{S} \Sigma^{\infty} Y \cong \Sigma^{\infty}(X \wedge Y) .
$$

For any $S$-module $M$ and any $\mathbb{L}$-spectrum $N, M \wedge_{\mathscr{L}} N$ is an $S$-module. In particular, $S \wedge \mathscr{L} N$ is an $S$-module for any $\mathbb{L}$-spectrum $N$.

We have the following categorical relationship between $\mathscr{S}[\mathbb{L}]$ and $\mathscr{M}_{S}$.

Lemma 5.3. The functor $S \wedge \mathscr{L}\left(\right.$ ?) $: \mathscr{S}[\mathbb{L}] \longrightarrow \mathscr{M}_{S}$ is left adjoint to the functor $F_{\mathscr{L}}(S, ?): \mathscr{M}_{S} \longrightarrow \mathscr{S}[\mathbb{L}]$ and right adjoint to the inclusion $\ell: \mathscr{M}_{S} \longrightarrow \mathscr{S}[\mathbb{L}]$.

This implies that to lift right adjoint functors from $\mathscr{S}[\mathbb{L}]$ to $\mathscr{M}_{S}$, we must first forget down to $\mathscr{S}[\mathbb{L}]$, next apply the given functor, and then apply the functor $S \wedge \mathscr{L}\left(\right.$ ?). For example, limits in $\mathscr{M}_{S}$ are created in this fashion.

Proposition 5.4. The category of $S$-modules is complete and cocomplete. Its colimits are created in $\mathscr{S}[\mathbb{L}]$. Its limits are created by applying the functor $S \wedge_{S}(?)$ to limits in $\mathscr{S}[\mathbb{L}]$. If $X$ is a based space and $M$ is an $S$-module, then $M \wedge X$ is an $S$-module, and the spectrum level cofibre of a map of $S$-modules is an $S$-module. For a based space $X$ and $S$-modules $M$ and $N$,

$$
\mathscr{M}_{S}(M \wedge X, N) \cong \mathscr{M}_{S}(M, S \wedge \mathscr{L} F(X, N)) .
$$


Moreover,

$$
M \wedge X \cong M \wedge_{S} \Sigma^{\infty} X \quad \text { and } \quad S \wedge_{\mathscr{L}} F(X, M) \cong F_{S}\left(\Sigma^{\infty} X, M\right) .
$$

Lemma 5.3 also explains our definition of function $S$-modules. Its second adjunction and the adjunction of Theorem 4.8 compose to give the adjunction displayed in the following theorem.

Theorem 5.5. The category $\mathscr{M}_{S}$ is symmetric monoidal under $\wedge_{S}$, and

$$
\mathscr{M}_{S}\left(M \wedge_{S} N, P\right) \cong \mathscr{M}_{S}\left(M, F_{S}(N, P)\right)
$$

for $S$-modules $M, N$, and $P$.

A homotopy in the category of $S$-modules is a map $M \wedge I_{+} \longrightarrow N$. A map of $S$-modules is a weak equivalence if it is a weak equivalence as a map of spectra. The derived category $\mathscr{D}_{S}$ of $S$-modules is constructed from the homotopy category $h \mathscr{M}_{S}$ by adjoining formal inverses to the weak equivalences; again, the process is made rigorous by CW approximation. We define sphere $S$-modules

$$
S_{S}^{n} \equiv S \wedge \mathscr{L} \mathbb{L} S^{n}
$$

and use them as the domains of attaching maps when defining cell and $\mathrm{CW} S$ modules. From here, the theory of cell and CW $S$-modules is exactly like the theory of cell and CW spectra and is obtained by specialization of the theory of cell $R$-modules to be discussed shortly. A weak equivalence of cell $S$-modules is a homotopy equivalence, any $S$-module is weakly equivalent to a $\mathrm{CW} S$-module, and $\mathscr{D}_{S}$ is equivalent to the homotopy category of $\mathrm{CW} S$-modules. Again, the $S$ module $S$ does not have the homotopy type of a $\mathrm{CW} S$-module. When working homotopically, we replace it with $S_{S} \equiv S_{S}^{0}$.

The following comparison between CW $S$-modules and CW $\mathbb{L}$-spectra establishes an equivalence between $\mathscr{D}_{S}$ and $\bar{h} \mathscr{S}[\mathbb{L}]$ and thus between $\mathscr{D}_{S}$ and $\bar{h} \mathscr{S}$. It is largely a recapitulation of results already discussed.

Theorem 5.6. The following conclusions hold.

(i) The functor $S \wedge \mathscr{L}(?): \mathscr{S}[\mathbb{L}] \longrightarrow \mathscr{M}_{S}$ carries $C W \mathbb{L}$-spectra to $C W S$ modules.

(ii) The forgetful functor $\mathscr{M}_{S} \longrightarrow \mathscr{S}[\mathbb{L}]$ carries $S$-modules of the homotopy types of $C W S$-modules to $\mathbb{L}$-spectra of the homotopy types of $C W \mathbb{L}$-spectra.

(iii) Every $C W S$-module $M$ is homotopy equivalent as an $S$-module to $S \wedge_{S} N$ for some $C W \mathbb{L}$-spectrum $N$.

(iv) The unit $\lambda: S \wedge \mathscr{L} M \longrightarrow M$ is a weak equivalence for all $\mathbb{L}$-spectra $M$ and is a homotopy equivalence of $\mathbb{L}$-spectra if $M$ has the homotopy type of a $C W \mathbb{L}$-spectrum. 
The functors $S \wedge \mathscr{L}(?)$ and the forgetful functor establish an adjoint equivalence between the stable homotopy category $\bar{h} \mathscr{S}[\mathbb{L}]$ and the derived category $\mathscr{D}_{S}$. This equivalence of categories preserves smash products and function spectra.

When doing classical homotopy theory, we can work interchangeably in any of the categories $\bar{h} \mathscr{S}, \bar{h} \mathscr{S}[\mathbb{L}]$, or $\mathscr{D}_{S}$. These three categories are equivalent, and the equivalences preserve all structure in sight. When working on the point set level, we have reached a nearly ideal situation with our construction of $\mathscr{M}_{S}$, and the rest of the article will describe how to exploit this.

\section{6. $S$-algebras and their Categories of modules}

Intuitively, $S$-algebras are as near to associative rings with unit as one can get in stable homotopy theory, and commutative $S$-algebras are as near as one can get to commutative rings.

Definition 6.1. An $S$-algebra is an $S$-module $R$ together with maps of $S$-modules $\eta: S \longrightarrow R$ and $\phi: R \wedge_{S} R \rightarrow R$ such that the following diagrams of $S$-modules commute:

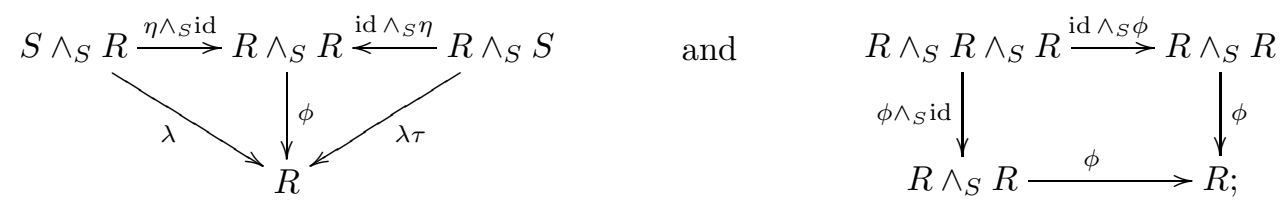

$R$ is commutative if the following diagram also commutes:

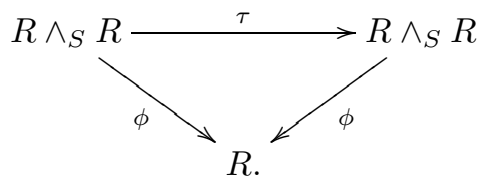

We shall not review the older definitions of $A_{\infty}$ and $E_{\infty}$ ring spectra. It turns out that they are equivalent to the structures that are given by the definition above, with the single exception that the unit map $\lambda$ of an $A_{\infty}$ or $E_{\infty}$ ring spectrum need not be an isomorphism. In other words, the natural ground category for $A_{\infty}$ and $E_{\infty}$ ring spectra is the category of $\mathbb{L}$-spectra rather than the category of $S$-modules. We state this formally.

Theorem 6.2. An $S$-algebra or commutative $S$-algebra is an $A_{\infty}$ or $E_{\infty}$ ring spectrum which is also an $S$-module. If $A$ is an $A_{\infty}$ ring spectrum, then $S \wedge \mathscr{L} A$ is a weakly equivalent $S$-algebra. If $A$ is an $E_{\infty}$ ring spectrum, then $S \wedge \mathscr{L} A$ is a weakly equivalent commutative $S$-algebra.

This means that we can use the older theory to construct examples. For example, the classical Thom spectra occur in nature as $E_{\infty}$ ring spectra, and [20] gives a 
machine for manufacturing $A_{\infty}$ and $E_{\infty}$ ring spectra from space level data. It shows that the Eilenberg-MacLane spectrum $H k$ of a ring $k$ is an $A_{\infty}$ ring spectrum and is an $E_{\infty}$ ring spectrum if $k$ is commutative and that the algebraic $K$-theory spectrum $K k$ of a commutative ring $k$ is an $E_{\infty}$ ring spectrum. Similarly, the spectra $k o$ and $k u$ that represent real and complex connective $K$-theory are $E_{\infty}$ ring spectra.

Since it is very convenient to have strict units, we shall always work with $S$ algebras.

Definition 6.3. Let $R$ be an $S$-algebra. A (left) $R$-module $M$ is an $S$-module together with a map $\mu: R \wedge_{S} M \rightarrow M$ of $S$-modules such that the following diagrams commute:

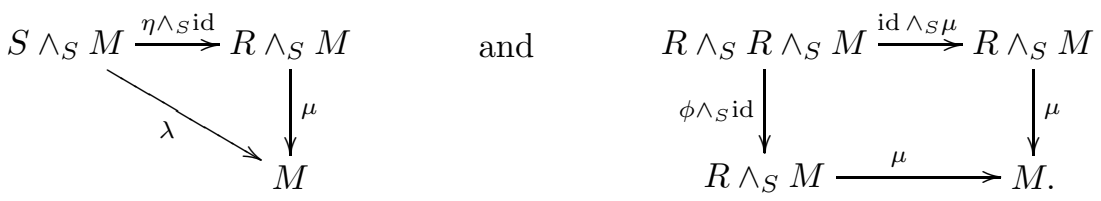

A map $f: M \longrightarrow M^{\prime}$ of $R$-modules is a map of $S$-modules such that the following diagram commutes:

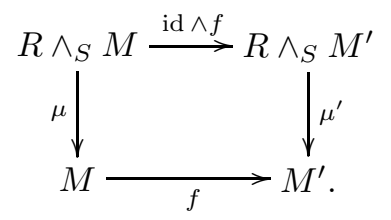

We let $\mathscr{M}_{R}$ denote the category of $R$-modules.

If $R$ is commutative, then an $R$-module is the same thing as a left module over $R$ regarded as an $S$-algebra, exactly as in algebra. From here, we can mimic vast areas of algebra, one particularly striking direction being the development of topological Hochschild homology. However, we shall concentrate on the generalized analog of stable homotopy theory that we obtain by studying the homotopy theory of $R$ modules for a fixed commutative $S$-algebra $R$. Everything that makes sense is also true in the non-commutative case.

Theorem 6.4. The category of $R$-modules is complete and cocomplete, with both limits and colimits created in the underlying category $\mathscr{M}_{S}$. Let $X$ be a based space, $K$ be an $S$-module, and $M$ and $N$ be $R$-modules. Then the following conclusions hold, where the displayed isomorphisms are obtained by restriction of the corresponding isomorphisms for $S$-modules.

(i) $M \wedge X$ is an $R$-module and the spectrum level cofibre of a map of $R$-modules is an $R$-module.

(ii) $S \wedge \mathscr{L} F(X, N)$ is an $R$-module and

$$
\mathscr{M}_{R}(M \wedge X, N) \cong \mathscr{M}_{R}(M, S \wedge \mathscr{L} F(X, N)) .
$$


(iii) $M \wedge_{S} K$ and $F_{S}(K, N)$ are $R$-modules and

$$
\mathscr{M}_{R}\left(M \wedge_{S} K, N\right) \cong \mathscr{M}_{R}\left(M, F_{S}(K, N)\right) .
$$

(iv) $F_{S}(M, K)$ is an $R$-module.

(v) As R-modules,

$$
M \wedge X \cong M \wedge_{S} \Sigma^{\infty} X \quad \text { and } \quad S \wedge \mathscr{L} F(X, N) \cong F_{S}\left(\Sigma^{\infty} X, N\right) .
$$

A homotopy in the category of $R$-modules is a map $M \wedge I_{+} \longrightarrow M^{\prime}$. A map of $R$-modules is a weak equivalence if it is a weak equivalence as a map of spectra. The derived category $\mathscr{D}_{R}$ is constructed from the homotopy category $h \mathscr{M}_{R}$ by adjoining formal inverses to the weak equivalences; again, the process is made rigorous by the approximation of general $R$-modules by cell $R$-modules.

Cell theory is based on the free $R$-module functor $\mathbb{F}_{R}$ from spectra to $R$-modules that is specified by $\mathbb{F}_{R} X=R \wedge_{S} \mathbb{F}_{S} X$, where $\mathbb{F}_{S} X=S \wedge \mathscr{L} \mathbb{L} X$. The term "free" is a slight misnomer, in view of the following result.

Proposition 6.5. The functor $\mathbb{F}_{R}: \mathscr{S} \longrightarrow \mathscr{M}_{R}$ is left adjoint to the functor that sends an $R$-module $M$ to the spectrum $F_{\mathscr{L}}(S, M)$, and there is a natural map of $R$-modules $\xi: \mathbb{F}_{R} M \longrightarrow M$ whose adjoint $M \longrightarrow F_{\mathscr{L}}(S, M)$ is a weak equivalence of spectra. Therefore

$$
\pi_{n}(M) \cong h \mathscr{M}_{R}\left(\mathbb{F}_{R} S^{n}, M\right) .
$$

In the stable homotopy category $\bar{h} \mathscr{S}, \mathbb{F}_{R} X$ is naturally isomorphic to the internal smash product $R \wedge X$ when $X$ is tame.

Thus $\mathbb{F}_{R}$ is left adjoint to a functor that is weakly equivalent to the obvious forgetful functor. This is the price to be paid for insisting on strict units, and it introduces no serious complications in the theory. Homotopically, the functor $\mathbb{F}_{R}$ behaves as one would expect. Generalizing (5.6), we define sphere $R$-modules by

$$
S_{R}^{n}=\mathbb{F}_{R} S^{n},
$$

and we use them as the domains of attaching maps when developing the cell theory of $R$-modules. For cells, we note that the cone functor $C E=E \wedge I$ commutes with $\mathbb{F}_{R}$, so that $C S_{R}^{n} \cong \mathbb{F}_{R} C S^{n}$. Thus, via the adjunction, maps out of sphere $R$-modules and their cones are induced by maps on the spectrum level. Using this, we can simply parrot the theory of cell spectra in the context of $R$-modules, reducing proofs to the spectrum level via adjunction. We easily obtain the Whitehead theorem for cell $R$-modules, and the approximation theorem to the effect that any $R$-module is weakly equivalent to a cell $R$-module. The category $\mathscr{D}_{R}$ is equivalent to the homotopy category of cell $R$-modules. If $R$ is connective, but not otherwise, we obtain the cellular approximation theorem when we restrict attention to $\mathrm{CW}$ 
$R$-modules, namely cell $R$-modules such that cells are only attached to cells of lower dimension.

The category $\mathscr{D}_{R}$ has all homotopy limits and colimits; the former are created as the corresponding constructions on the underlying diagrams of spectra; the latter require application of the functor $S \wedge \mathscr{L}($ ?). Thus we have enough information to quote the categorical form of Brown's representability theorem given in [6]. Adams' analog [3] for functors defined only on finite CW spectra also applies in our context, with the same proof.

Theorem 6.6 (Brown). A contravariant functor $k: \mathscr{D}_{R} \rightarrow$ Sets is representable in the form $k(M) \cong \mathscr{D}_{R}(M, N)$ for some $R$-module $N$ if and only if $k$ converts wedges to products and converts homotopy pushouts to weak pullbacks.

Theorem 6.7 (Adams). A contravariant group-valued functor $k$ on the homotopy category of finite cell $R$-modules is representable in the form $k(M) \cong \mathscr{D}_{R}(M, N)$ for some $R$-module $N$ if and only if $k$ converts finite wedges to direct products and converts homotopy pushouts to weak pullbacks of underlying sets.

In fact, Brown's theorem is the kind of formal result that can be derived in any (closed) model category in the sense of Quillen (see [8] for a good exposition), and we have the following result. Serre fibrations of spectra are maps that satisfy the covering homotopy property with respect to the set of cone spectra

$$
\left\{\Sigma_{q}^{\infty} C S^{n} \mid q \geq 0 \text { and } n \geq 0\right\} .
$$

Relative cell $R$-modules $M \longrightarrow N$ are constructed exactly like cell $R$-modules, except that one starts the inductive construction of $N=\cup N_{n}$ with $N_{0}=M$.

We write $q$-cofibrations and $q$-fibrations here to avoid confusion with cofibrations (HEP) and fibrations (CHP); the ambiguous use of the same term for both the classical and the model theoretic concepts is one of the banes of the literature.

Theorem 6.8. The category of $R$-modules is a model category. Its weak equivalences are the maps of $R$-modules that are weak equivalences of spectra. Its $q$ cofibrations are the retracts of relative cell $R$-modules. Its q-fibrations are the maps $M \longrightarrow N$ such that $F_{\mathscr{L}}(S, M) \longrightarrow F_{\mathscr{L}}(S, N)$ is a Serre fibration of spectra.

\section{The Smash product of $R$-modules and function $R$-MOdules}

Continuing to work with our fixed commutative $S$-algebra $R$, we mimic the definition of tensor products of modules over algebras.

Definition 7.1. For $R$-modules $M$ and $N$, define $M \wedge_{R} N$ to be the coequalizer displayed in the following diagram of $S$-modules:

$$
M \wedge_{S} R \wedge_{S} N \underset{\text { id } \wedge_{S} \nu}{\stackrel{\mu \wedge_{S} \text { id }}{\longrightarrow}} M \wedge_{S} N \longrightarrow M \wedge_{R} N .
$$


where $\mu$ and $\nu$ are the given actions of $R$ on $M$ and $N$. Then $M \wedge_{R} N$ has a canonical $R$-module structure induced from the $R$-module structure of $M$ or, equivalently, $N$.

Of course, $S$ is a commutative $S$-algebra and our new $M \wedge_{S} N$ coincides with our old $M \wedge_{S} N$. The functor $\wedge_{R}$ preserves colimits in each of its variables, and smash products with spaces commute with $\wedge_{R}$, in the sense that

$$
X \wedge\left(M \wedge_{R} N\right) \cong(X \wedge M) \wedge_{R} N
$$

Therefore the functor $\wedge_{R}$ commutes with cofibre sequences in each of its variables. We have analogous relations with smash products over $S$ and an adjunction that can be thought of as completing Proposition 5.4.

Proposition 7.2. For an $S$-module $K$,

$$
K \wedge_{S}\left(M \wedge_{R} N\right) \cong\left(K \wedge_{S} M\right) \wedge_{R} N
$$

and

$$
\mathscr{M}_{S}\left(M \wedge_{R} N, K\right) \cong \mathscr{M}_{R}\left(N, F_{S}(M, K)\right) .
$$

The associativity, commutativity, and unity of the smash product over $S$ is inherited by the smash product over $R$.

Theorem 7.3. Under the smash product over $R$, the category of $R$-modules is symmetric monoidal with unit $R$.

We can deduce not only formal but also homotopical properties of $\wedge_{R}$ from corresponding properties of $\wedge_{S}$. As in Section 4 , we use an isomorphism of universes $f: U \oplus U \rightarrow U$ to define the internal smash product $f_{*}(E \wedge F)$.

Proposition 7.4. Let $X$ and $Y$ be spectra and let $N$ be an $R$-module. There is a natural isomorphism of $R$-modules

$$
\mathbb{F}_{R} X \wedge_{R} N \cong \mathbb{F}_{S} X \wedge_{S} N
$$

There is also a natural isomorphism of $R$-modules

$$
\mathbb{F}_{R} X \wedge_{R} \mathbb{F}_{R} Y \cong \mathbb{F}_{R} f_{*}(X \wedge Y) .
$$

If $M$ and $N$ are cell $R$-modules, then $M \wedge_{R} N$ is a cell $R$-module with one $(p+q)$-cell for each p-cell of $M$ and $q$-cell of $N$.

Theorem 7.5. If $M$ is a cell $R$-module and $\phi: N \longrightarrow N^{\prime}$ is a weak equivalence of $R$-modules, then

$$
\operatorname{id} \wedge_{R} \phi: M \wedge_{R} N \longrightarrow M \wedge_{R} N^{\prime}
$$

is a weak equivalence of $R$-modules. 
We construct $\wedge_{R}$ as a functor $\mathscr{D}_{R} \times \mathscr{D}_{R} \rightarrow \mathscr{D}_{R}$ by approximating one of the variables by a cell $R$-module.

We have a function spectrum functor $F_{R}$ to go with our smash product. It is defined as the equalizer of a certain pair of maps $F_{S}(M, N) \longrightarrow F_{S}\left(R \wedge_{S} M, N\right)$. The details are dictated by the expected adjunction. Again, $F_{R}(M, N)$ inherits a structure of $R$-module from $M$ or, equivalently, $N$.

Proposition 7.6. For $R$-modules $N$ and $P$ and an $S$-module $K$,

$$
\mathscr{M}_{R}\left(K \wedge_{S} N, P\right) \cong \mathscr{M}_{S}\left(K, F_{R}(N, P)\right) .
$$

If $M$ is an $R$-module, then

$$
\mathscr{M}_{R}\left(M \wedge_{R} N, P\right) \cong \mathscr{M}_{R}\left(M, F_{R}(N, P)\right) .
$$

Therefore

$$
F_{R}\left(M \wedge_{R} N, P\right) \cong F_{R}\left(M, F_{R}(N, P)\right) .
$$

Formal arguments from the adjunction, as in algebra, give a natural associative and unital composition pairing

$$
\pi: F_{R}(N, P) \wedge_{R} F_{R}(M, N) \longrightarrow F_{R}(M, P) .
$$

Parenthetically, we note that this gives rise to a host of examples of $S$-algebras; in fact, $R$ itself need not be commutative in the following result.

Proposition 7.7. For an $R$-module $M, F_{R}(M, M)$ is an $S$-algebra; For $R$-modules $M$ and $N, F_{R}(M, N)$ is an $\left(F_{R}(N, N), F_{R}(M, M)\right)$-bimodule.

Proposition 7.8. Let $X$ be a spectrum and $M$ be an $R$-module. There is a natural isomorphism of $R$-modules

$$
F_{R}\left(\mathbb{F}_{R} X, M\right) \cong F_{S}\left(\mathbb{F}_{S} X, M\right) .
$$

The functor $F_{R}(M, N)$ converts colimits and cofibre sequences in $M$ to limits and fibre sequences. It preserves limits and fibre sequences in $N$. Using the previous result to deal with sphere $R$-modules, we obtain the analog of Theorem 7.5.

Proposition 7.9. If $M$ is a cell $R$-module and $\phi: N \longrightarrow N^{\prime}$ is a weak equivalence of $R$-modules, then

$$
F_{R}(\mathrm{id}, \phi): F_{R}(M, N) \longrightarrow F_{R}\left(M, N^{\prime}\right)
$$

is a weak equivalence of $R$-modules.

In the derived category $\mathscr{D}_{R}, F_{R}(M, N)$ means $F_{R}(\Gamma M, N)$, where $\Gamma M$ is a cell approximation of $M$.

Summarizing, we obtain the following derived category level conclusion. 
Theorem 7.10. The derived category $\mathscr{D}_{R}$ is symmetric monoidal under the product derived from $\wedge_{R}$, and

$$
\mathscr{D}_{R}\left(M \wedge_{R} N, P\right) \cong \mathscr{D}_{R}\left(M, F_{R}(N, P)\right) .
$$

There is a formal theory of duality (explained in [16, Ch. III]) that now applies to $\mathscr{D}_{R}$. We define the dual of an $R$-module $M$ to be $D_{R} M=F_{R}(M, R)$. We have an evaluation map $\varepsilon: D_{R} M \wedge_{R} M \longrightarrow R$ and a map $\eta: R \rightarrow F_{R}(M, M)$, namely the adjoint of $\lambda: R \wedge_{R} M \longrightarrow M$. There is also a natural map

$$
\nu: F_{R}(L, M) \wedge_{R} N \longrightarrow F_{R}\left(L, M \wedge_{R} N\right) .
$$

By composition with $F_{R}($ id, $\lambda), \nu$ specializes to a map

$$
\nu: D_{R} M \wedge_{R} M \longrightarrow F_{R}(M, M) .
$$

We say that $M$ is "strongly dualizable" if it has a coevaluation map $\bar{\eta}: R \longrightarrow$ $M \wedge_{R} D_{R} M$ such that the following diagram commutes in $\mathscr{D}_{R}$ :

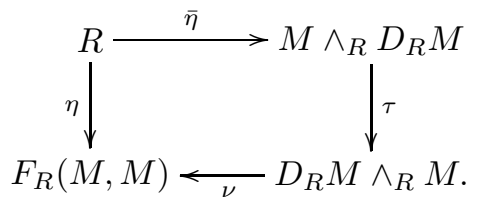

The definition has many purely formal implications. The map $\nu$ of (7.12) is an isomorphism in $\mathscr{D}_{R}$ if either $L$ or $N$ is strongly dualizable. The map $\nu$ of (7.13) is an isomorphism if and only if $M$ is strongly dualizable, and the coevaluation map $\bar{\eta}$ is then the composite $\tau \nu^{-1} \eta$ in (7.14). The natural map

$$
\rho: M \longrightarrow D_{R} D_{R} M
$$

is an isomorphism if $M$ is strongly dualizable. The natural map

$$
\wedge: F_{R}(M, N) \wedge_{R} F_{R}\left(M^{\prime}, N^{\prime}\right) \longrightarrow F_{R}\left(M \wedge_{R} M^{\prime}, N \wedge_{R} N^{\prime}\right)
$$

is an isomorphism if $M$ and $M^{\prime}$ are strongly dualizable or if $M$ is strongly dualizable and $N=R$.

Say that a cell $R$-module $N$ is a wedge summand up to homotopy of a cell $R$ module $M$ if there is a homotopy equivalence of $R$-modules between $M$ and $N \vee N^{\prime}$ for some cell $R$-module $N^{\prime}$. We say that $N$ is semi-finite if it is a wedge summand up to homotopy of a finite cell $R$-module. In contrast with the usual stable homotopy category, a semi-finite $R$-module need not have the homotopy type of a finite cell $R$-module.

Theorem 7.11. A cell R-module is strongly dualizable if and only if it is semifinite.

The analogy with finitely generated projective modules in algebra should be clear. 


\section{TOR AND Ext IN TOPOLOGY AND ALGEBRA}

Still restricting for definiteness to a commutative $S$-algebra $R$ and its modules, we define Tor and Ext groups as the homotopy groups of derived smash product and function modules.

Definition 8.1. For $R$-modules $M$ and $N$, define

$$
\operatorname{Tor}_{n}^{R}(M, N)=\pi_{n}\left(M \wedge_{R} N\right)
$$

and

$$
\operatorname{Ext}_{R}^{n}(M, N)=\pi_{-n}\left(F_{R}(M, N)\right) .
$$

Note that $\operatorname{Tor}_{*}^{R}(M, N)$ and $\operatorname{Ext}_{R}^{*}(M, N)$ are $\pi_{*}(R)$-modules.

We emphasize that the smash product and function spectra are understood to be taken in the derived category $\mathscr{D}_{R}$. At this point in our exposition, we act as traditional topologists, taking it for granted that all spectra and modules are to be approximated as cell modules, without change of notation, whenever necessary. Various properties reminiscent of those of the classical Tor and Ext functors follow directly from the definition and the results of the previous sections. The intuition is that the definition gives an analogue of the differential Tor and Ext functors (alias hyperhomology and cohomology functors) in the context of differential graded modules over differential graded algebras. In particular, the grading should not be thought of as the resolution grading of the classical torsion product, but rather as a total grading that sums a resolution degree and an internal degree; this idea will be made precise by the grading of the spectral sequences that we shall describe for the calculation of these functors.

Proposition 8.2. $\operatorname{Tor}_{*}^{R}(M, N)$ satisfies the following properties.

(i) If $R, M$, and $N$ are connective, then $\operatorname{Tor}_{n}^{R}(M, N)=0$ for $n<0$.

(ii) A cofibre sequence $N^{\prime} \rightarrow N \rightarrow N^{\prime \prime}$ gives rise to a long exact sequence

$\cdots \rightarrow \operatorname{Tor}_{n}^{R}\left(M, N^{\prime}\right) \rightarrow \operatorname{Tor}_{n}^{R}(M, N) \rightarrow \operatorname{Tor}_{n}^{R}\left(M, N^{\prime \prime}\right) \rightarrow \operatorname{Tor}_{n-1}^{R}\left(M, N^{\prime}\right) \rightarrow \cdots$.

(iii) $\operatorname{Tor}_{*}^{R}(M, R) \cong \pi_{*}(M)$ and, for a spectrum $X$,

$$
\operatorname{Tor}_{*}^{R}(M, \mathbb{F} X) \cong \pi_{*}(M \wedge X) .
$$

(iv) The functor $\operatorname{Tor}_{*}^{R}(M, ?)$ carries wedges to direct sums.

The commutativity and associativity relations for the smash product imply various further properties. We content ourselves with the following examples:

$$
\operatorname{Tor}_{*}^{R}(M, N) \cong \operatorname{Tor}_{*}^{R}(N, M)
$$

and

$$
\operatorname{Tor}_{*}^{R}\left(M \wedge_{R} N, P\right) \cong \operatorname{Tor}_{*}^{R}\left(M, N \wedge_{R} P\right) .
$$

Say that a spectrum $N$ is coconnective if $\pi_{n}(N)=0$ for $n>0$. 
Proposition 8.3. $\operatorname{Ext}_{R}^{*}(M, N)$ satisfies the following properties.

(i) If $R$ and $M$ are connective and $N$ is coconnective, then $\operatorname{Ext}_{R}^{n}(M, N)=0$ for $n<0$.

(ii) Fibre sequences $N^{\prime} \rightarrow N \rightarrow N^{\prime \prime}$ and cofibre sequences $M^{\prime} \rightarrow M \rightarrow M^{\prime \prime}$ give rise to long exact sequences

$$
\cdots \rightarrow \operatorname{Ext}_{R}^{n}\left(M, N^{\prime}\right) \rightarrow \operatorname{Ext}_{R}^{n}(M, N) \rightarrow \operatorname{Ext}_{R}^{n}\left(M, N^{\prime \prime}\right) \rightarrow \operatorname{Ext}_{R}^{n+1}\left(M, N^{\prime}\right) \rightarrow \cdots
$$

and

$\cdots \rightarrow \operatorname{Ext}_{R}^{n}\left(M^{\prime \prime}, N\right) \rightarrow \operatorname{Ext}_{R}^{n}(M, N) \rightarrow \operatorname{Ext}_{R}^{n}\left(M^{\prime}, N\right) \rightarrow \operatorname{Ext}_{R}^{n+1}\left(M^{\prime \prime}, N\right) \rightarrow \cdots$.

(iii) $\operatorname{Ext}_{R}^{*}(R, N) \cong \pi_{-*}(N)$ and, for a spectrum $X$,

$$
\operatorname{Ext}_{R}^{*}(\mathbb{F} X, N) \cong \pi_{-*}(F(X, N)) .
$$

(iv) The functor $\operatorname{Ext}_{R}^{*}(?, N)$ carries wedges to products.

Passing to homotopy groups from the pairings (7.7), we obtain the following further property. As usual, for a spectrum $E$, abbreviate

$$
E_{n}=\pi_{n}(E)=E^{-n} .
$$

Proposition 8.4. There is a natural, associative, and unital system of pairings of $R^{*}$-modules

$$
\pi^{*}: \operatorname{Ext}_{R}^{*}(M, N) \otimes_{R^{*}} \operatorname{Ext}_{R}^{*}(L, M) \longrightarrow \operatorname{Ext}_{R}^{*}(L, N) .
$$

The formal duality theory of the previous section implies the following result, together with various other such isomorphisms.

Proposition 8.5. For a finite cell $R$-module $M$ and any $R$-module $N$,

$$
\operatorname{Tor}_{n}^{R}\left(D_{R} M, N\right) \cong \operatorname{Ext}_{R}^{-n}(M, N) .
$$

Thinking of the derived category $\mathscr{D}_{R}$ as a stable homotopy category, we may change notations and reinterpret the functors Tor and Ext as prescribing homology and cohomology theories in this category.

Definition 8.6. Let $M$ and $E$ be $R$-modules. Define

$$
E_{n}^{R}(M)=\pi_{n}\left(E \wedge_{R} M\right) \text { and } E_{R}^{n}(M)=\pi_{-n}\left(F_{R}(M, E)\right) .
$$

The properties of Tor and Ext translate directly to statements about homology and cohomology. All of the standard homotopical machinery is available to us, and the previous result now takes the form of Spanier-Whitehead duality.

Corollary 8.7. For a finite cell $R$-module $M$ and any $R$-module $E$,

$$
E_{n}^{R}\left(D_{R} M\right) \cong E_{R}^{-n}(M) .
$$


Since the equivalence between the classical stable homotopy category and the derived category of $S$-modules preserves smash products and function spectra, we obtain all of the usual homology and cohomology theories by taking $R=S$.

We also obtain the classical algebraic Tor and Ext groups as special cases, by specialization to Eilenberg-Mac Lane spectra. Thus let $R$ be a discrete commutative ring for a moment. Recall that $H R$ denotes a spectrum whose zeroth homotopy group is $R$ and whose remaining homotopy groups are zero. It follows from multiplicative infinite loop space theory [20] that the Eilenberg-MacLane spectrum $H R=K(R, 0)$ is an $E_{\infty}$ ring spectrum. Analogously, if $M$ is an $R$-module, then $H M$ can be constructed as an $H R$-module. We shall see a quick and easy construction shortly. Granting this, we have the following result.

Theorem 8.8. For a discrete commutative ring $R$ and $R$-modules $M$ and $N$,

$$
\operatorname{Tor}_{*}^{R}(M, N) \cong \operatorname{Tor}_{*}^{H R}(H M, H N)
$$

and

$$
\operatorname{Ext}_{R}^{*}(M, N) \cong \operatorname{Ext}_{H R}^{*}(H M, H N) \text {. }
$$

Under the second isomorphism, the topologically defined pairing

$$
\operatorname{Ext}_{H R}^{*}(H M, H N) \otimes_{R} \operatorname{Ext}_{H R}^{*}(H L, H M) \longrightarrow \operatorname{Ext}_{H R}^{*}(H L, H N)
$$

coincides with the algebraic Yoneda product.

The proof is clear enough: we just check the axioms for Tor and Ext.

We can elaborate this result to an equivalence of derived categories. Recall from [28] or $\left[15\right.$, Ch.III] that the derived category $\mathscr{D}_{R}$ is obtained from the homotopy category of chain complexes over $R$ by formally inverting the quasi-isomorphisms, exactly as we obtained the category $\mathscr{D}_{H R}$ from the homotopy category of $H R$ modules by inverting the weak equivalences. The algebraic theory of cell and $\mathrm{CW}$ chain complexes over $R$ in the latter source makes the analogy precise and gives a treatment of tensor products and Hom functors in $\mathscr{D}_{R}$ that exactly parallels our treatment of $\wedge_{H R}$ and $F_{H R}$. The proof of the equivalence is quite easy. The category $\mathscr{D}_{H R}$ is equivalent to the homotopy category of $\mathrm{CW} H R$-modules and cellular maps. It is a simple matter to see that CW $H R$-modules have associated chain complexes. This gives a functor $\mathscr{D}_{H R} \longrightarrow \mathscr{D}_{R}$. An inverse functor $\Phi$ is obtained by applying Brown's representability theorem. Indeed, for a given chain complex $X$, the functor $k$ on $\mathscr{D}_{H R}$ specified by $k(M)=\mathscr{D}_{R}\left(C_{*}(M), X\right)$ satisfies the hypotheses of that result, and we let $\Phi(X)$ represent this functor. Specialization to $R$-modules regarded as chain complexes concentrated in degree zero gives the promised construction of Eilenberg-MacLane $H R$-modules from $R$-modules. 
Theorem 8.9. The cellular chain functor $C_{*}$ on $H R$-modules induces an equivalence of categories $\mathscr{D}_{H R} \longrightarrow \mathscr{D}_{R}$. The functor $C_{*}$ satisfies $H_{*}\left(C_{*}(M)\right) \cong \pi_{*}(M)$ and carries the functors $\wedge_{H R}$ and $F_{H R}$ to the functors $\otimes_{R}$ and $\operatorname{Hom}_{R}$. The inverse equivalence $\Phi$ satisfies $\pi_{*}(\Phi(X)) \cong H_{*}(X)$ and carries the functors $\otimes_{R}$ and $\operatorname{Hom}_{R}$ to the functors $\wedge_{H R}$ and $F_{H R}$.

Proof. By construction, we have an adjunction

$$
\mathscr{D}_{R}\left(C_{*}(M), X\right) \cong \mathscr{D}_{H R}(M, \Phi(X)),
$$

and one checks that its unit and counit are isomorphisms. The statements relating $\wedge_{H R}$ and $F_{H R}$ to $\otimes_{R}$ and $\operatorname{Hom}_{R}$ are all consequences of the fact that if $M$ and $N$ are CW $H R$-modules, then $M \wedge_{H R} N$ is a CW $H R$-module such that

$$
C_{*}\left(M \wedge_{H R} N\right) \cong C_{*}(M) \otimes_{R} C_{*}(N) .
$$

\section{Universal COefFicient AND KÜNneth SPECTRAL SEQUenCES}

Returning to our general commutative $S$-algebra $R$, we find spectral sequences for the calculation of our Tor and Ext groups that are analogous to the EilenbergMoore (or hyperhomology) spectral sequences in differential homological algebra. Compare $[9,13,15]$. They may be viewed as giving universal coefficient and Künneth spectral sequences for homology and cohomology theories on $R$-modules, and they specialize to give such spectral sequences for homology and cohomology theories on spectra.

Theorem 9.1. For $R$-modules $M$ and $N$, there are natural spectral sequences of differential $R_{*}$-modules

$$
E_{p, q}^{2}=\operatorname{Tor}_{p, q}^{R_{*}}\left(M_{*}, N_{*}\right) \Longrightarrow \operatorname{Tor}_{p+q}^{R}(M, N)
$$

and

$$
E_{2}^{p, q}=\operatorname{Ext}_{R^{*}}^{p, q}\left(M^{*}, N^{*}\right) \Longrightarrow \operatorname{Ext}_{R}^{p+q}(M, N) .
$$

Moreover, the pairing $F_{R}(M, N) \wedge_{R} F_{R}(L, M) \rightarrow F_{R}(L, N)$ induces a pairing of spectral sequences that coincides with the algebraic Yoneda pairing

$$
\operatorname{Ext}_{R^{*}}^{*, *}\left(M^{*}, N^{*}\right) \otimes_{R^{*}} \operatorname{Ext}_{R^{*}}^{*, *}\left(L^{*}, M^{*}\right) \longrightarrow \operatorname{Ext}_{R^{*}}^{*, *}\left(L^{*}, N^{*}\right)
$$

on the $E_{2}$-level and that converges to the induced pairing of Ext groups.

The Tor spectral sequence is of standard homological type, with

$$
d_{p, q}^{r}: E_{p, q}^{r} \longrightarrow E_{p-r, q+r-1}^{r} .
$$

It lies in the right half-plane, and it converges strongly. The Ext spectral sequence is of standard cohomological type, with

$$
d_{r}: E_{r}^{p, q} \rightarrow E_{r}^{p+r, q-r+1} .
$$


It lies in the right half plane. In the language of Boardman [5] (see also [12, App $\mathrm{B}]$ ), it is conditionally convergent. It therefore converges strongly if, for each fixed $(p, q)$, there are only finitely many $r$ such that $d_{r}$ is non-zero on $E_{r}^{p, q}$.

Setting $M=\mathbb{F}_{R} X$ in the two spectral sequences of Theorem 8.1, we obtain a universal coefficient spectral sequence. We have written the stars to indicate the way the grading is usually thought of in cohomology.

Theorem 9.2 (Universal coefficient). For an $R$-module $N$ and any spectrum $X$, there are spectral sequences of the form

$$
\operatorname{Tor}_{*, *}^{R_{*}}\left(R_{*}(X), N_{*}\right) \Longrightarrow N_{*}(X)
$$

and

$$
\operatorname{Ext}_{R^{*}}^{*, *}\left(R_{-*}(X), N^{*}\right) \Longrightarrow N^{*}(X)
$$

Of course, replacing $R$ and $N$ by Eilenberg-Mac Lane spectra $H R$ and $H N$ for a ring $R$ and $R$-module $N$, we obtain the classical universal coefficient theorems. Replacing $N$ by $\mathbb{F}_{R} Y$ and by $F_{R}\left(\mathbb{F}_{R} Y, R\right)$ in the two universal coefficient spectral sequences, we arrive at Künneth spectral sequences.

Theorem 9.3 (Künneth). For any spectra $X$ and $Y$, there are spectral sequences of the form

$$
\operatorname{Tor}_{*, *}^{R_{*}}\left(R_{*}(X), R_{*}(Y)\right) \Longrightarrow R_{*}(X \wedge Y)
$$

and

$$
\operatorname{Ext}_{R^{*}}^{* * *}\left(R_{-*}(X), R^{*}(Y)\right) \Longrightarrow R^{*}(X \wedge Y) .
$$

Adams [1] first observed that one can derive Künneth spectral sequences from universal coefficient spectral sequences, and he observed that, by duality, the four spectral sequences of Theorems 9.2 and 9.3 imply two more universal coefficient and two more Künneth spectral sequences. He derived spectral sequences of this sort under the hypothesis that his given ring spectrum $E$ is the colimit of finite subspectra $E_{\alpha}$ such that $H^{*}\left(E_{\alpha} ; E^{*}\right)$ is $E^{*}$-projective and the Atiyah-Hirzebruch spectral sequence converging from $H^{*}\left(E_{\alpha} ; E^{*}\right)$ to $E^{*}\left(E_{\alpha}\right)$ satisfies $E_{2}=E_{\infty}$. Of course, this is an ad hoc calculational hypothesis that requires case-by-case verification. It covers some cases that are not covered by the results above, and conversely. The cited paper of Adams, and his later book [2], are prime sources for the first flowering of stable homotopy theory. While some of their foundational parts may be obsolete, their applications and calculational parts certainly are not.

The following generalized Künneth theorem admits a number of variants; see [11].

Theorem 9.4. Let $E$ and $R$ be commutative $S$-algebras and $M$ and $N$ be $R$ modules. Then there is a spectral sequence of differential $E_{*}(R)$-modules of the 
form

$$
\operatorname{Tor}_{p, q}^{E_{*}(R)}\left(E_{*}(M), E_{*}(N)\right) \Longrightarrow E_{p+q}\left(M \wedge_{R} N\right) \text {. }
$$

10. Algebraic constructions in the Derived Category of $R$-modules

If we replace the pair $(S, R)$ by a pair $(R, A)$ in Definition 6.1 , we arrive at the notion of an algebra $A$ over a commutative $S$-algebra $R$. For example, the $S$-algebras $F_{R}(M, M)$ of Proposition 7.8 are actually $R$-algebras. Again, if $A$ is an algebra over a discrete commutative ring $R$, then $H A$ is an $H R$-algebra. Proceeding in this line, we can, for instance, construct $R$-modules whose homotopy groups realize the Hochschild homology of $A$ with coefficients in $(A, A)$-bimodules.

However, we now proceed in a more homotopical direction, thinking of the derived category of $R$-modules as an analog of the stable homotopy category. From this point of view, we have the notion of an $R$-ring spectrum, which is just like the classical notion of a ring spectrum in the stable homotopy category.

Definition 10.1. An $R$-ring spectrum $A$ is an $R$-module $A$ with unit $\eta: R \longrightarrow A$ and product $\phi: A \wedge_{R} A \longrightarrow A$ in $\mathscr{D}_{R}$ such that the following left and right unit diagram commutes in $\mathscr{D}_{R}$.

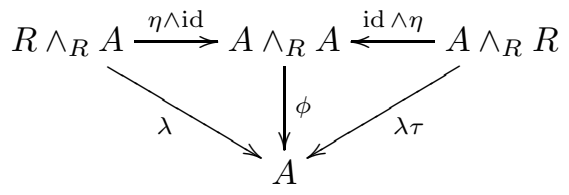

$A$ is associative or commutative if the appropriate diagram commutes in $\mathscr{D}_{R}$.

Lemma 10.2. If $A$ and $B$ are $R$-ring spectrum, then so is $A \wedge_{R} B$. If $A$ and $B$ are associative or commutative, then so is $A \wedge_{R} B$.

By neglect of structure, an $R$-ring spectrum $A$ is a ring spectrum in the sense of classical stable homotopy theory; its unit is the composite of the unit of $R$ and the unit of $A$ and its product is the composite of the product of $A$ and the canonical map

$$
A \wedge A \simeq A \wedge_{S} A \longrightarrow A \wedge_{R} A .
$$

Similarly, for an $R$-algebra $A$, we have the evident homotopical notion of an $A$ module spectrum. These structures play a role in the study of $\mathscr{D}_{R}$ analogous to the role played by ring spectra and their module spectra in classical stable homotopy theory. When $R=S, S$-ring spectra and their module spectra are equivalent to classical ring spectra and their module spectra.

We show in this section how to construct quotients $M / I M$ and localizations $M\left[Y^{-1}\right]$ of modules over a commutative $S$-algebra $R$ and indicate in the next section when these constructions inherit a structure of $R$-ring spectrum from an $R$-ring 
spectrum structure on $M$. When specialized to $M U$, these results give highly structured versions of spectra that in the past were constructed by means of the Baas-Sullivan theory of manifolds with singularities or the Landweber exact functor theorem. At least at odd primes, the results give an entirely satisfactory, and very simple, treatment of $M U$-ring structures on the resulting $M U$-modules.

We are interested in homotopy groups, and we make use of the isomorphisms

$$
M_{n}=h \mathscr{S}\left(S^{n}, M\right) \cong h \mathscr{M}_{S}\left(S_{S}^{n}, M\right) \cong h \mathscr{M}_{R}\left(S_{R}^{n}, M\right)
$$

to represent elements as maps of $R$-modules. For $x \in R_{n}$, the composite map of $R$-modules

$$
S_{R}^{n} \wedge_{R} M \stackrel{x \wedge \mathrm{id}}{\longrightarrow} R \wedge_{R} M \stackrel{\lambda}{\longrightarrow} M
$$

is a module theoretic version of the map $x \cdot: \Sigma^{n} M \longrightarrow M$, and we agree to write $\Sigma^{n} M$ for $S_{R}^{n} \wedge_{R} M$ in this section. By Proposition 7.4, $S_{R}^{n} \wedge_{R} M$ is isomorphic as an $R$-module to $S_{S}^{n} \wedge_{S} M$ and, by Theorem $4.7, S_{S}^{n} \wedge_{S} M$ is weakly equivalent as a spectrum to $S^{n} \wedge M$. Therefore, the $R$-module $\Sigma^{n} M$ is a model for the spectrum level suspension of $M$.

Definition 10.3. Define $M / x M$ to be the cofibre of the map (9.4) and let $\rho$ : $M \longrightarrow M / x M$ be the canonical map. Inductively, for a finite sequence $\left\{x_{1}, \ldots, x_{n}\right\}$ of elements of $R_{*}$, define

$$
M /\left(x_{1}, \ldots, x_{n}\right) M=N / x_{n} N, \text { where } N=M /\left(x_{1}, \ldots, x_{n-1}\right) M .
$$

For a (countably) infinite sequence $X=\left\{x_{i}\right\}$, define $M / X M$ to be the telescope of the $M /\left(x_{1}, \ldots, x_{n}\right) M$, where the telescope is taken with respect to the successive canonical maps $\rho$.

Clearly we have a long exact sequence

$$
\cdots \longrightarrow \pi_{q-n}(M) \stackrel{x \cdot}{\longrightarrow} \pi_{q}(M) \stackrel{\rho_{*}}{\longrightarrow} \pi_{q}(M / x M) \longrightarrow \pi_{q-n-1}(M) \longrightarrow \cdots .
$$

If $x$ is not a zero divisor for $\pi_{*}(M)$, then $\rho_{*}$ induces an isomorphism of $R_{*}$-modules

$$
\pi_{*}(M) / x \cdot \pi_{*}(M) \cong \pi_{*}(M / x M) .
$$

If $\left\{x_{1}, \ldots, x_{n}\right\}$ is a regular sequence for $\pi_{*}(M)$, in the sense that $x_{i}$ is not a zero divisor for $\pi_{*}(M) /\left(x_{1}, \ldots, x_{i-1}\right) \pi_{*}(M)$ for $1 \leq i \leq n$, then

$$
\pi_{*}(M) /\left(x_{1}, \ldots, x_{n}\right) \pi_{*}(M) \cong \pi_{*}\left(M /\left(x_{1}, \ldots, x_{n}\right) M\right),
$$

and similarly for a possibly infinite regular sequence $X=\left\{x_{i}\right\}$. The following result implies that $M / X M$ is independent of the ordering of the elements of the set $X$. We write $R / X$ instead of $R / X R$. 
Lemma 10.4. For a set $X$ of elements of $R_{*}$, there is a natural weak equivalence

$$
(R / X) \wedge_{R} M \longrightarrow M / X M
$$

In particular, for a finite set $X=\left\{x_{1}, \ldots, x_{n}\right\}$,

$$
R /\left(x_{1}, \ldots, x_{n}\right) \simeq\left(R / x_{1}\right) \wedge_{R} \cdots \wedge_{R}\left(R / x_{n}\right) .
$$

If $I$ denotes the ideal generated by $X$, then it is reasonable to define

$$
M / I M=M / X M
$$

However, this notation must be used with caution since, if we fail to restrict attention to regular sequences $X$, the homotopy type of $M / X M$ will depend on the set $X$ and not just on the ideal it generates. For example, quite different modules are obtained if we repeat a generator $x_{i}$ of $I$ in our construction.

We next construct localizations of $R$-modules at countable multiplicatively closed subsets $Y$ of $R_{*}$. Let $\left\{y_{i}\right\}$ be any cofinal sequence of $Y$, with $y_{i} \in R_{n_{i}}$, so that every $y \in Y$ divides some $y_{i}$. We may represent $y_{i}$ by an $R$-map $S_{R}^{0} \longrightarrow S_{R}^{-n_{i}}$, which we also denote by $y_{i}$. Let $q_{0}=0$ and, inductively, $q_{i}=q_{i-1}+n_{i}$. The $R$-map

$$
S_{R}^{0} \wedge_{R} M \stackrel{y_{i} \wedge \text { id }}{\longrightarrow} S_{R}^{-n_{i}} \wedge_{R} M
$$

represents $y_{i}$. Smashing over $R$ with $S_{R}^{-q_{i-1}}$, we obtain a sequence of $R$-maps

$$
S_{R}^{-q_{i-1}} \wedge_{R} M \longrightarrow S_{R}^{-q_{i}} \wedge_{R} M
$$

Definition 10.5. Define the localization of $M$ at $Y$, denoted $M\left[Y^{-1}\right]$, to be the telescope of the sequence of maps (10.11). Since $M \cong S_{R}^{0} \wedge_{R} M$ in $\mathscr{D}_{R}$, we may regard the inclusion of the initial stage $S_{R}^{0} \wedge_{R} M$ of the telescope as a natural map $\lambda: M \longrightarrow M\left[Y^{-1}\right]$.

Since homotopy groups commute with localization, we see immediately that $\lambda$ induces an isomorphism of $R_{*}$-modules

$$
\pi_{*}\left(M\left[Y^{-1}\right]\right) \cong \pi_{*}(M)\left[Y^{-1}\right] .
$$

As in Lemma 10.9, the localization of $M$ is the smash product of $M$ with the localization of $R$.

Lemma 10.6. For a multiplicatively closed set $Y$ of elements of $R_{*}$, there is a natural equivalence

$$
R\left[Y^{-1}\right] \wedge_{R} M \longrightarrow M\left[Y^{-1}\right]
$$

Moreover, $R\left[Y^{-1}\right]$ is independent of the ordering of the elements of $Y$. For sets $X$ and $Y, R\left[(X \cup Y)^{-1}\right]$ is equivalent to the composite localization $R\left[X^{-1}\right]\left[Y^{-1}\right]$. 


\section{1. $R$-RING STRUCTURES ON LOCALIZATIONS AND ON QUOTIENTS BY IDEALS}

The behavior of localizations with respect to $R$-ring structures is immediate.

Proposition 11.1. Let $Y$ be a multiplicatively closed set of elements of $R_{*}$. If $A$ is an $R$-ring spectrum, then $A\left[Y^{-1}\right]$ is an $R$-ring spectrum such that $\lambda: A \longrightarrow A\left[Y^{-1}\right]$ is a map of $R$-ring spectra. If $A$ is associative or commutative, then so is $A\left[Y^{-1}\right]$.

Proof. By Lemmas 10.2 and 10.14, it suffices to observe that $R\left[Y^{-1}\right]$ is an associative and commutative $R$-ring spectrum with unit $\lambda$ and product the equivalence

$$
R\left[Y^{-1}\right] \wedge_{R} R\left[Y^{-1}\right] \simeq R\left[Y^{-1}\right]\left[Y^{-1}\right] \simeq R\left[Y^{-1}\right]
$$

This doesn't work for quotients since $(R / X) / X$ is not equivalent to $R / X$. However, we can analyze the problem by analyzing the deviation, and, by Lemma 10.9, we may as well work one element at a time. We have a necessary condition for $R / x$ to be an $R$-ring spectrum that is familiar from classical stable homotopy theory.

Lemma 11.2. Let $A$ be an $R$-ring spectrum. If $A / x A$ admits an $R$-ring spectrum structure such that $\rho: A \longrightarrow A / x A$ is a map of $R$-ring spectra, then $x: A / x A \longrightarrow$ $A / x A$ is null homotopic as a map of $R$-modules.

Thus, for example, the Moore spectrum $S / 2$ is not an $S$-ring spectrum since the map $2: S / 2 \longrightarrow S / 2$ is not null homotopic. To give a criterion for when $R / x$ does have an $R$-ring spectrum structure, we first note an easy formal lemma.

Lemma 11.3. Let $\rho: R \longrightarrow M$ be any map of $R$-modules. Then

$$
(\rho \wedge \mathrm{id}) \circ \rho \simeq(\mathrm{id} \wedge \rho) \circ \rho: R \longrightarrow M \wedge_{R} M
$$

Theorem 11.4. Let $x \in R_{m}$ and assume that $\pi_{m+1}(R / x)=0$ and $\pi_{2 m+1}(R / x)=$ 0 . Then $R / x$ admits a structure of $R$-ring spectrum with unit $\rho: R \longrightarrow R / x$. Therefore $A / X A$ admits a structure of $R$-ring spectrum such that $\rho: A \longrightarrow A / X A$ is a map of $R$-ring spectra for every $R$-ring spectrum $A$ and every sequence $X$ of elements of $R_{*}$ such that $\pi_{m+1}(R / x)=0$ and $\pi_{2 m+1}(R / x)=0$ if $x \in X$ has degree $m$. 
Proof. Consider the following diagram in the derived category $\mathscr{D}_{R}$ :

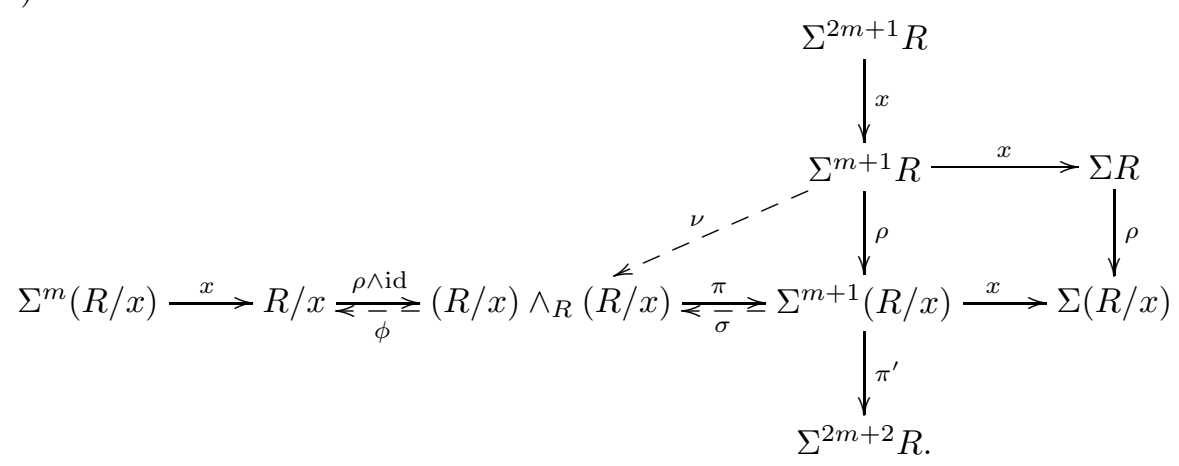

The map $x$ is that specified by (10.4). The bottom row is the cofibre sequence that results from the equivalence

$$
(R / x) \wedge_{R}(R / x) \simeq(R / x) / x
$$

of Lemma 10.9, and the column is also a cofibre sequence. The composite $x \circ \rho$ is null homotopic since $\rho \circ x$ is null homotopic and the square commutes. Therefore there is a map $\nu$ such that $\pi \circ \nu=\rho$, and $\nu$ is unique since $\pi_{m+1}(R / x)=0$. Since $\pi \circ \nu \circ x=\rho \circ x=0, \nu \circ x$ factors through a map $\Sigma^{2 m+1} R \longrightarrow R / x$. Since $\pi_{2 m+1}(R / x)=0$, such maps are null homotopic. Thus $\nu \circ x$ is null homotopic. Therefore there is a map $\sigma$ such that $\sigma \circ \rho=\nu$. Now $\pi \circ \sigma \circ \rho=\pi \circ \nu=\rho$, hence $(\pi \circ \sigma-\mathrm{id}) \rho=0$. Therefore $\pi \circ \sigma-$ id factors through a map $\Sigma^{2 m+2} R \longrightarrow \Sigma^{m+1}(R / x)$. Again, such maps are null homotopic. Therefore $\pi \circ \sigma=\mathrm{id}$. Thus the bottom cofibre sequence splits (proving in passing that $x: \Sigma^{m}(R / x) \longrightarrow R / x$ is null homotopic, as it must be). A choice $\phi$ of a splitting gives a product on $R / x$. The unit condition $\phi \circ(\rho \wedge \mathrm{id})=\mathrm{id}$ is automatic. To see that $\phi \circ(\mathrm{id} \wedge \rho)=\mathrm{id}$, we observe that, by the lemma,

$$
(\phi \circ(\mathrm{id} \wedge \rho)-\mathrm{id}) \circ \rho=\phi \circ(\mathrm{id} \wedge \rho-\rho \wedge \text { id }) \circ \rho=0 .
$$

Therefore $\phi \circ($ id $\wedge \rho)$ - id factors through a map $\Sigma^{m+1} R \longrightarrow R / x$. Again, such maps are null homotopic, hence $\phi \circ(\mathrm{id} \wedge \rho)=\mathrm{id}$. This completes the proof that $R / x$ is an $R$-ring spectrum with unit $\rho$. The rest follows from Lemmas 10.9 and 10.2 .

The product on $R / x$ can be described a little more concretely. The wedge sum

$$
(\rho \wedge \mathrm{id}) \vee \sigma:(R / x) \vee \Sigma^{m+1}(R / x) \longrightarrow(R / x) \wedge_{R}(R / x)
$$

is an equivalence. The product $\phi$ restricts to the identity on the first wedge summand and to the trivial map on the second wedge summand. Thus the product is determined by the choice of $\sigma$, and two choices of $\sigma$ differ by a composite

$$
\Sigma^{m+1}(R / x) \stackrel{\pi^{\prime}}{\longrightarrow} \Sigma^{2 m+2} R \longrightarrow(R / x) \wedge_{R}(R / x) .
$$


By the splitting (10.6) and the assumption that $\pi_{m+1}(R / x)=0$, we can view the second map as an element of $\pi_{2 m+2}(R / x)$. If $x$ is not a zero divisor, then $\pi_{*}^{\prime}=0$ on homotopy groups and any two products have the same effect on homotopy groups.

For an $R$-ring spectrum $A$ and an element $x$ as in the theorem, we give $A / x A \simeq$ $(R / x) \wedge_{R} A$ the product induced by one of our constructed products on $R / x$ and the given product on $A$. We refer to any such product as a "canonical" product on $A / x A$. Observe that, by first using the product on $A$, the product on $A / x A$ can be factored through

$$
\phi \wedge_{R} \text { id }:(R / x) \wedge_{R}(R / x) \wedge_{R} A \longrightarrow(R / x) \wedge_{R} A .
$$

This allows us to smash any diagram giving information about the product on $R / x$ with $A$ and so obtain information about the product on $A / x A$. Obviously any diagram so constructed is a diagram of right $A$-modules via the product action of $A$ on itself. This smashing with $A$ can kill obstructions. Clearly, a map of $A$-modules $\Sigma^{q} A \longrightarrow M$ is determined by its restriction $S^{q} \longrightarrow M$ along the unit of $A$ regarded as a map of spectra (or $S$-modules), which is just an element of $\pi_{q}(M)$. This leads to the following result.

Theorem 11.5. Let $x \in R_{m}$ and assume that $\pi_{m+1}(R / x)=0$ and $\pi_{2 m+1}(R / x)=$ 0 . Let $A$ be an $R$-ring spectrum and assume that $\pi_{2 m+2}(A / x A)=0$. Then there is a unique canonical product on $A / x A$. If $A$ is commutative, then $A / x A$ is commutative. If $A$ is associative and $\pi_{3 m+3}(A / x A)=0$, then $A / x A$ is associative.

Proof. The second arrow of (11.7) becomes zero after smashing with $A$ since it is then given by an element of $\pi_{2 m+2}(A / x A)=0$. This proves the uniqueness statement. The commutativity statement follows since if $\phi$ is a canonical product on $A / x A$, then so is $\phi \tau$. The associativity statement requires consideration of the restriction of the iterated product to the wedge summands of $A / x A \wedge_{R} A / x A \wedge_{R}$ $A / x A$. The details are similar to, but simpler than, those in the proof of Theorem 11.4 .

Iterating and observing that passage to telescopes can kill obstructions, we arrive at the following fundamental conclusion.

Theorem 11.6. Assume that $R_{i}=0$ if $i$ is odd. Let $X$ be a sequence of non zero divisors in $R_{*}$ such that $\pi_{*}(R / X)$ is concentrated in degrees congruent to zero mod 4. Then $R / X$ has a unique canonical structure of $R$-ring spectrum, and it is commutative and associative.

\section{The specialization to $M U$-modules and $M U$-ring SPeCtra}

The classical Thom spectra arise in nature as $E_{\infty}$ ring spectra. In fact, it was inspection of their prespectrum level definition in terms of Grassmannians that 
first led to the theory of $E_{\infty}$ ring spectra [19]. Applying the functor $S \wedge_{\mathscr{L}}(?)$, we obtain models for Thom spectra which are commutative $S$-algebras. Of course, the homotopy groups of $M U$ are concentrated in even degrees, and every non-zero element is a non zero divisor. Thus the results above have the following immediate corollary.

Theorem 12.1. Let $X$ be a regular sequence in $M U_{*}$, let $I$ be the ideal generated by $X$, and let $Y$ be any sequence in $M U_{*}$. Then there is an $M U$-ring spectrum $(M U / X)\left[Y^{-1}\right]$ and a natural map of $M U$-ring spectra (the unit map)

$$
\eta: M U \longrightarrow(M U / X)\left[Y^{-1}\right]
$$

such that

$$
\eta_{*}: M U_{*} \longrightarrow \pi_{*}\left((M U / X)\left[Y^{-1}\right]\right)
$$

realizes the natural homomorphism of $M U_{*}$-algebras

$$
M U_{*} \longrightarrow\left(M U_{*} / I\right)\left[Y^{-1}\right] .
$$

If $M U_{*} / I$ is concentrated in degrees congruent to zero mod 4, then there is a unique canonical product on $(M U / X)\left[Y^{-1}\right]$, and this product is commutative and associative.

In comparison with earlier constructions of this sort based on the Baas-Sullivan theory of manifolds with singularities or on Landweber's exact functor theorem (where it applies), we have obtained a simpler proof of a substantially stronger result. We emphasize that an $M U$-ring spectrum is a much richer structure than just a ring spectrum and that commutativity and associativity in the $M U$-ring spectrum sense are much more stringent conditions than mere commutativity and associativity of the underlying ring spectrum.

We illustrate by explaining how $B P$ appears in this context. Fix a prime $p$ and write $(?)_{p}$ for localization at $p$. Let $B P$ be the Brown-Peterson spectrum at $p$. We are thinking of Quillen's idempotent construction [24], and we have the splitting maps $i: B P \longrightarrow M U_{p}$ and $e: M U_{p} \longrightarrow B P$. These are maps of commutative and associative ring spectra such that $e \circ i=$ id. Let $I$ be the kernel of the composite

$$
M U_{*} \longrightarrow M U_{p *} \longrightarrow B P_{*} .
$$

Then $I$ is generated by a regular sequence $X$, and our $M U / X$ is a canonical integral version of $B P$. For the moment, let $B P^{\prime}=(M U / X)_{p}$. Let $\xi: B P \longrightarrow B P^{\prime}$ be the composite

$$
B P \stackrel{i}{\longrightarrow} M U_{p} \stackrel{\eta_{p}}{\longrightarrow} B P^{\prime} .
$$

It is immediate that $\xi$ is an equivalence. In effect, since we have arranged that $\eta_{p}$ has the same effect on homotopy groups as $e, \xi$ induces the identity map of 
$\left(M U_{*} / I\right)_{p}$ on homotopy groups. By the splitting of $M U_{p}$ and the fact that selfmaps of $M U_{p}$ are determined by their effect on homotopy groups [2, II.9.3], maps $M U_{p} \longrightarrow B P$ are determined by their effect on homotopy groups. This implies that $\xi \circ e=\eta_{p}: M U_{p} \longrightarrow B P^{\prime}$. The product on $B P$ is the composite

$$
B P \wedge B P \stackrel{i \wedge i}{\longrightarrow} M U_{p} \wedge M U_{p} \stackrel{\phi}{\longrightarrow} M U_{p} \stackrel{e}{\longrightarrow} B P .
$$

Since $\eta_{p}$ is a map of $M U$-ring spectra and thus of ring spectra, a trivial diagram chase now shows that the equivalence $\xi: B P \longrightarrow B P^{\prime}$ is a map of ring spectra.

We conclude that our $B P^{\prime}$ is a model for $B P$ that is an $M U$-ring spectrum, commutative and associative if $p>2$. The situation for $p=2$ is interesting. We conclude from the equivalence that $B P^{\prime}$ is commutative and associative as a ring spectrum, although we do not know that it is commutative or associative as an $M U$-ring spectrum.

Recall that $\pi_{*}(B P)=\mathbb{Z}_{(p)}\left[v_{i} \mid \operatorname{deg}\left(v_{i}\right)=2\left(p^{i}-1\right)\right]$, where the generators $v_{i}$ come from $\pi_{*}(M U)$ (provided that we use the Hazewinkel generators). We list a few of the spectra derived from $B P$, with their coefficient rings. Let $\mathbb{F}_{p}$ denote the field with $p$ elements.

$$
\begin{array}{llll}
B P\langle n\rangle & \mathbb{Z}_{(p)}\left[v_{1}, \ldots, v_{n}\right] & E(n) & \mathbb{Z}_{(p)}\left[v_{1}, \ldots, v_{n}, v_{n}^{-1}\right] \\
P(n) & \mathbb{F}_{p}\left[v_{n}, v_{n+1}, \ldots\right] & B(n) & \mathbb{F}_{p}\left[v_{n}^{-1}, v_{n}, v_{n+1}, \ldots\right] \\
k(n) & \mathbb{F}_{p}\left[v_{n}\right] & K(n) & \mathbb{F}_{p}\left[v_{n}, v_{n}^{-1}\right]
\end{array}
$$

By the method just illustrated, we can construct canonical integral versions of the $B P\langle n\rangle$ and $E(n)$. All of these spectra fit into the context of Theorem 11.1. If $p>2$, they all have unique canonical commutative and associative $M U$-ring spectrum structures. Further study is needed when $p=2$. In any case, this theory makes it unnecessary to appeal to Baas-Sullivan theory or to Landweber's exact functor theorem for the construction and analysis of spectra such as these.

With more sophisticated techniques, the second author [14] has proven that BP can be constructed as a commutative $S$-algebra, and in fact admits uncountably many distinct such structures. There is much other ongoing work on the construction and application of new commutative $S$-algebras, by Hopkins, Miller, McClure, and others, and we have recently proven that the periodic $K$-theory spectra $K O$ and $K U$ can be constructed as commutative $S$-algebras. The enriched multiplica-

tive structures on rings and modules that we have discussed are rapidly becoming a standard tool in the study of periodicity phenomena in stable homotopy theory.

\section{REFERENCES}

[1] J.F. Adams. Lectures on generalised cohomology. Springer Lecture Notes in Mathematics Vol. 99, 1969, 1-138.

[2] J.F. Adams. Stable homotopy and generalized homology. University of Chicago. 1974, 1994. 
[3] J.F. Adams. A variant of E.H.Brown's representability theorem. Topology, 10 (1971), 185198.

[4] J.M. Boardman. Stable homotopy theory. Thesis, Warwick 1964; mimeographed notes from Warwick and Johns Hopkins Universities, 1965-1970.

[5] J.M. Boardman. Conditionally convergent spectral sequences. Preprint. 1981.

[6] E.H. Brown, Jr. Abstract homotopy theory. Trans. Amer. Math. Soc. 119 (1965), 79-85.

[7] R.R. Bruner, J.P. May, J.E. McClure, and M. Steinberger. $H_{\infty}$ ring spectra and their applications. Springer Lecture Notes in Mathematics Vol. 1176. 1986.

[8] W.G. Dwyer and J. Spalinski. Homotopy theories and model categories. This volume.

[9] S. Eilenberg and J.C. Moore. Homology and fibrations, I. Comm. Math. Helv. 40 (1966), 199-236.

[10] A.D. Elmendorf, J.P.C. Greenlees, I. Kriz, and J.P. May. Commutative algebra in stable homotopy theory and a completion theorem. Mathematical Research Letters 1 (1994), 225239.

[11] A.D. Elmendorf, I. Kriz, M. A. Mandell, and J.P. May. Rings, modules, and algebras in stable homotopy theory. In preparation.

[12] J.P.C. Greenlees and J.P. May. Generalized Tate cohomology. Memoirs Amer. Math. Soc. To appear.

[13] V.K.A.M. Gugenheim and J.P. May. On the theory and applications of differential torsion products. Memoirs Amer. Math. Soc. 142, 1974.

[14] I. Kriz. Towers of $E_{\infty}$ ring spectra with an application to BP. Preprint. 1993.

[15] I. Kriz, and J.P. May. Operads, algebras, modules, and motives. Astérisque. To appear.

[16] L.G. Lewis, Jr., J.P. May, and M. Steinberger (with contributions by J.E. McClure). Equivariant stable homotopy theory. Springer Lecture Notes in Mathematics Vol. 1213. 1986.

[17] S. Mac Lane. Categories for the Working Mathematician. Springer-Verlag. 1971.

[18] J.P. May. The Geometry of Iterated Loop Spaces. Springer Lecture Notes in Mathematics Vol. 271. 1972.

[19] J.P. May (with contributions by N. Ray, F. Quinn and J. Tornehave). $E_{\infty}$ ring spaces and $E_{\infty}$ ring spectra. Springer Lecture Notes in Mathematics Vol. 577. 1977.

[20] J.P. May. Multiplicative infinite loop space theory. J. Pure and Applied Algebra, 26 (1982), 1-69.

[21] J.P. May. Derived categories in algebra and topology. In the Proceedings of the Eleventh International Conference on Topology, Rendiconti dell Istituto Matematico dell Università di Trieste. To appear.

[22] J.P. May. Equivariant homotopy and cohomology theory. CBMS Lectures. In preparation.

[23] D.G. Quillen. Homotopical algebra. Springer Lecture Notes in Mathematics Vol. 43. 1967.

[24] D.G. Quillen. On the formal group laws of unoriented and complex cobordism theory. Bull. Amer. Math. Soc. 75 (1969), 1293-1298.

[25] A. Robinson. Derived tensor products in stable homotopy theory. Topology 22 (1983), 1-18.

[26] A. Robinson. Spectra of derived module homomorphisms. Math. Proc. Camb. Phil. Soc. 101 (1987), 249-257.

[27] A. Robinson. The extraordinary derived category. Math. Z. 196(1987), 231-238.

[28] J.L. Verdier. Catégories dérivées. Springer Lecture Notes in Mathematics Vol. 569, 1977, $262-311$.

[29] G.W. Whitehead. Elements of homotopy theory. Springer-Verlag. 1978.

Purdue University Calumet, Hammond, IN 46323 USA

E-mail address: aelmendo@@math.purdue.edu

The University of Michigan, Ann Arbor, MI 48109-1003 USA

E-mail address: ikriz@@ath.lsa.umich.edu

The University of Chicago, Chicago, IL 60637 USA

E-mail address: mandell@@math.uchicago.edu

The University of Chicago, Chicago, IL 60637 USA

E-mail address: may@@math.uchicago.edu 